\title{
STEPS TOWARD DETERMINATION OF THE SIZE AND STRUCTURE OF THE BROAD-LINE REGION IN ACTIVE GALACTIC NUCLEI. I. AN 8 MONTH CAMPAIGN OF MONITORING NGC 5548 WITH IUE
}

J. Clavel, ${ }^{1}$ G. A. Reichert, ${ }^{2}$ D. Alloin, ${ }^{3}$ D. M. Crenshaw, ${ }^{2}$ G. Kriss, ${ }^{4}$ J. H. Krolik, ${ }^{4}$ M. A. Malkan, ${ }^{5}$ H. Netzer, ${ }^{6}$ B. M. Peterson, ${ }^{7}$ W. Wamsteker, ${ }^{1}$ A. Altamore, ${ }^{8}$ T. Baribaud, ${ }^{3}$ P. Barr, ${ }^{9}$ S. Beck, ${ }^{6}$ L. Binette, ${ }^{10}$ G. E. Bromage, ${ }^{11}$ N. Brosch, ${ }^{6}$ A. I. Diaz, ${ }^{12}$ A. V. Filippenko, ${ }^{13}$ K. Fricke, ${ }^{14}$ C. M. Gaskell, ${ }^{15}$ P. Giommi, ${ }^{9}$ I. S. Glass, ${ }^{16}$ P. GondhaleKar, ${ }^{11}$ R. L. Hackney, ${ }^{17}$ J. P. HalPern, ${ }^{18}$ D. J. HutTer, ${ }^{2}$ S. Jörsäter, ${ }^{19}$ A. L. KinNey, ${ }^{20}$ W. Kollatschny, ${ }^{14}$ A. KoratKar, ${ }^{15}$ K. T. Korista, ${ }^{7}$ A. LaOr, ${ }^{6}$ J. P. Lasota, ${ }^{3}$ E. Leibowitz, ${ }^{6}$ D. MaOz, ${ }^{6}$ P. G. Martin, ${ }^{10}$ T. MAZeh, ${ }^{6}$ E. J. A. Meurs, ${ }^{21}$ A. D. NaIr, ${ }^{22}$ P. O'Brien, ${ }^{23}$

D. Pelat, ${ }^{3}$ E. Perez, ${ }^{24}$ G. C. Perola, ${ }^{8}$ R. L. Ptak, ${ }^{22}$ P. Rodriguez-Pascual, ${ }^{25}$

E. I. Rosenblatt, ${ }^{26}$ A. C. Sadun, ${ }^{27}$ M. Santos-Lleo, ${ }^{25}$ R. A. Shaw, ${ }^{2}$ P. S. SMith, ${ }^{28}$ G. M. StirPe, ${ }^{29}$ R. StONer, ${ }^{22}$ W. H. Sun, ${ }^{30}$ M.-H. Ulrich, ${ }^{31}$ E. van Groningen, ${ }^{32}$ AND W. ZhenG ${ }^{33}$ Received 1990 January 22 ; accepted 1990 June 27

\begin{abstract}
We present emission-line and ultraviolet continuum observations of a type I Seyfert galaxy in which the time resolution is adequate for describing the character of variability. Using the IUE satellite, the nucleus of NGC 5548 was observed every 4 days for a period of 8 months. Its mean properties - continuum shape, line ratios - are not unusual for type I Seyfert galaxies, but it was found to be strongly variable. The ultraviolet continuum flux and broad emission line fluxes varied significantly, going through three large maxima and three deep minima. The ratio of maximum to minimum flux was $\simeq 4.5$ for the continuum at $1350 \AA$, and the continuum was significantly bluer when it was brighter. The high-ionization emission lines showed the strongest variations, with $\mathrm{N}$ v $\lambda 1240$ and He II $\lambda 1640$ exhibiting maximum-to-minimum flux ratios as high as those of the continuum. Intermediate-ionization lines, including Ly $\alpha \lambda 1216, \mathrm{C}$ IV $\lambda 1549$, and C III] $\lambda 1909$, had maximum-to-minimum amplitudes of $\sim 2$, and $\mathrm{Mg}$ II 22798 , the lowest ionization line, exhibited the smallest amplitude fluctuations, $\sim 1.3$. The great majority of all variations were well resolved in time. Apart from $\mathrm{Mg}$ II $\lambda 2798$, the emission-line variations correlate extremely well with those of the $1350 \AA$ continuum if allowance is made for a systematic delay, lending qualitative support to the view that photoionization by the nuclear con-
\end{abstract}

${ }^{1}$ ESA IUE Observatory, P.O. Box 50727, 28080 Madrid, Spain.

2 Astronomy Program, Computer Sciences Corporation, Goddard Space Flight Center, Code 684.9, Building 21, Greenbelt, MD 20771.

3 Observatoire de Paris, 92195 Meudon Principal Cedex, France.

4 The Johns Hopkins University, Department of Physics and Astronomy, Baltimore, MD 21218.

5 University of California, Department of Astronomy, Los Angeles, CA 90024-1562.

${ }^{6}$ School of Physics and Astronomy and Wise Observatory, Tel-Aviv University, Tel Aviv 69978, Israel.

7 Department of Astronomy, Ohio State University, 174 West 18th Avenue, Columbus, OH 43210.

8 Istituto Astronomico dell'Università, Via Lancisi 29, I-00161 Rome, Italy.

9 EXOSAT Observatory, Astrophysics Division of ESA, ESTEC, Postbus 299, 2200-AG Noordwijk, The Netherlands.

${ }^{\circ}$ CITA, McLennan Laboratories, University of Toronto, 60 St. George Street, Toronto, Ontario, Canada M5S 1A1.

${ }_{11}$ Astrophysics Group, Rutherford Appleton Laboratory, Chilton, Didcot, Oxfordshire OX11 0QX, United Kingdom.

12 Departamento de Física Teórica, C-XI, Universidad Autónoma, Cantoblanco, 28049 - Madrid, Spain.

13 Department of Astronomy, University of California at Berkeley, Berkeley, CA 94720.

14 Universitätssternwarte, Geismarlandstrasse 11, D-3400 Göttingen, Germany.

15 University of Michigan, Astronomy Department, Dennison Building, Ann Arbor, MI 48109-1090.

${ }^{16}$ South African Astronomical Observatory, P.O. Box 9, Observatory, 7935 South Africa.

17 Western Kentucky University, Department of Physics and Astronomy, Bowling Green, KY 42101.

18 Columbia University, Department of Physics, 538 West 120th Street, New York, NY 10027.

19 ESA STECF, Karl-Schwarzschild-Strasse 2, D-8046 Garching-bei-München, Germany.

${ }^{20}$ Space Telescope Science Institute, 3700 San Martin Drive, Baltimore, MD 21218.

21 Max-Planck-Institut für Extraterrestrische Physik, Giessenbachstrasse, D-80416 Garching-bei-München, Germany.

22 Bowling Green State University, Physics and Astronomy Department, Bowling Green, OH 43403.

${ }^{23}$ Department of Astronomy, University College London, Gower Street, London WC1E 6BT, United Kingdom.

24 Instituto de Astrofísica de Canarias, 38200 La Laguna, Tenerife, Spain.

${ }^{25}$ Instituto de Astronomia, Universidad Complutense, 28040 Madrid 3, Spain.

26 University of California at San Diego, CASS, Code C-011, La Jolla, CA 92093.

27 Bradley Observatory, Agnes Scott College, Decatur, GA 30030.

${ }_{28}$ Steward Observatory, University of Arizona, Tucson, AZ 85721.

${ }^{29}$ Osservatorio Astronomico di Bologna, Via Zamboni 33, I-40126 Bologna, Italy.

30 Laboratory for Astronomy and Solar Physics, NASA/GSFC, Greenbelt, MD 20771.

31 European Southern Observatory, Karl-Schwarzschild-Strasse 2, D-8046 Garching-bei-München, Germany.

32 Uppsala Astronomical Observatory, Box 515, S-75120 Uppsala, Sweden.

${ }^{33}$ University of Alabama, Department of Physics and Astronomy, Tuscaloosa, AL 35487-0324. 
tinuum is responsible for driving the emission lines. The delay of a given line seems to depend on the degree of ionization of its species. The He II $\lambda 1640$ and $N$ V $\lambda 1240$ features exhibit the shortest delay, $\Delta t \sim 4-10$ days, while the Ly $\alpha \lambda 1216$ and $\mathrm{C}$ IV $\lambda 1549$ lines yield 8-16 days. The Si IV $+\mathrm{O}$ IV] $\lambda 1402$ feature and the $\mathrm{C}$ III] $\lambda 1909$ line exhibit significantly larger delays, between 12 and 34 days. In the case of $\mathrm{Mg}$ II $\lambda 2798$, the crosscorrelation is broad and shallow, so that the delay is only loosely constrained, $\Delta t \sim 34-72$ days.

Subject headings: galaxies: individual (NGC 5548) - galaxies: nuclei - galaxies: Seyfert ultraviolet: spectra

\section{INTRODUCTION}

For more than 20 years it has been known that the fluxes of the continuum and broad emission lines of active galactic nuclei (AGNs) are variable (Andrillat and Souffrin 1968; Dibai and Pronik 1967; Fitch, Pacholczyk, and Weymann 1967). It was also realized quite early that the broad emission line and continuum variations are closely related (Cherepashchuk and Lyutyi 1973), as would be expected if the gas responsible for these lines is photoionized and heated by the continuum source. If photoionization is indeed the correct explanation for the emission lines' source of energy, the local response to changes in the continuum flux takes less than $1 \mathrm{hr}$, but the line-emitting region is predicted to be much larger-light-days to many light-years across - scaling crudely as $L^{1 / 2}$, where $L$ is the ionizing luminosity of the nucleus (Davidson and Netzer 1979). Therefore, one would expect emission-line fluctuations to follow continuum fluctuations, but spread out and delayed by an amount comparable to the light-travel time across the emission-line region.

In principle, it is possible to deconvolve the emission-line and continuum light curves to infer the detailed structure of the emission-line region (Blandford and McKee 1982; Capriotti, Foltz, and Peterson 1982). It might be possible to obtain by this method the geometrical configuration and the run of physical conditions of the broad-line region (BLR) gas as a function of distance from the central source. Such a deconvolution would amount to a direct test of the size prediction made by photoionization models. Not surprisingly, therefore, much observational effort has been recently devoted to the spectrophotometric monitoring of active galaxies (see, e.g., the review by Peterson $1988 a$ and references therein). The best-studied sources so far are, in order of decreasing luminosity, Fairall 9 (Clavel, Wamsteker, and Glass 1989; Koratkar and Gaskell 1989), Akn 120 (Peterson, Korista, and Wagner 1989), Mrk 279 (Maoz et al. 1990), NGC 5548 (Netzer et al. 1990; Wamsteker et al. 1990; Peterson et al. 1990b; Rosenblatt and Malkan 1990), NGC 4151 (Ulrich et al. 1984; Gaskell and Sparke 1986; Clavel et al. 1987). However, all of these studies have suffered from at least one of the following problems: inadequate temporal resolution, insufficient span of observations, too many gaps in the time series, or low ratio of fluctuation amplitude to measurement uncertainty. In the case of the optical studies, the interpretation is also hampered by the considerable difference in photon energy between the continuum band monitored (frequently the $B$-band) and the continuum band which actually drives the lines (13 eV to $\sim 5 \mathrm{keV}$, depending on the individual line).

We therefore initiated a program whose object was to circumvent all these problems. The choice of the target (NGC 5548) was mainly driven by practical considerations. A large apparent brightness, a long IUE observability window (8 months), and a documented history of strong line and continuum variability (e.g., Gregory, Ptak, and Stoner 1982; Barr, Willis, and Wilson 1983; Peterson et al. 1990b; Wamsteker et al. 1990) were the main selection criteria. Regular observations are essential to obtain a good description of the power spectrum of fluctuations, and are an absolute prerequisite to carrying out the deconvolution program outlined by Blandford and McKee (1982). The sampling interval of 4 days was determined by examination of previous observations and by comparison with a rough estimate from photoionization modeling of the light-travel time across the broad emission line region. We chose to observe in the ultraviolet to solve other problems. The observed continuum is closer in wavelength to the ionizing continuum driving the strong emission lines, and there is little dilution of the observed continuum by stellar light. The UV emission lines dominate the cooling of the broad-line clouds, and they span a wide range of ionization states. Moreover, by observing from space we were able to achieve greater photometric accuracy and eliminate interruptions to the observing schedule posed by weather or lunar phase. However, in order to maximize the scientific return from this effort, a parallel ground-based monitoring program was initiated (Peterson $1988 b$ ). Its results will be reported separately (Peterson et al. $1990 a$ ).

NGC 5548 is a low-redshift $(z=0.0174)$ Seyfert I galaxy. In the radio band, it shows a weak compact nuclear source straddled by two lobes with a total extent of $4.4 \mathrm{kpc}$ (Wilson and Ulvestad 1982). Deep [O III] $\lambda 5007$ exposures reveal an extended $(4.0 \mathrm{kpc} \times 2.7 \mathrm{kpc})$ emission-line region roughly aligned with the radio structure (Wilson et al. 1989). NGC 5548 was detected out to $100 \mu \mathrm{m}$ by the IRAS satellite (Edelson and Malkan 1986). In X-rays it qualifies as a low-luminosity AGN, with $L_{2-10 \mathrm{keV}}=4.5 \times 10^{43} \mathrm{ergs}^{-1}$ (Turner and Pounds 1989; Kaastra and Barr 1989). Branduardi-Raymont (1988) reported a possible correlation between the variations at $\mathrm{X}$-ray, UV, and optical wavelengths. This last author also presented evidence of $X$-ray spectral variations, the $2-10 \mathrm{keV}$ spectrum becoming systematically softer when the source brightens. These spectral changes may be related to the existence of a "soft X-ray excess" which dominates the emission below $1 \mathrm{keV}$ (Turner and Pounds 1989). A strong correlation between the ultraviolet and the optical variations is indicated by the results of Wamsteker et al. (1990), with the continuum becoming slightly bluer when brighter. Netzer et al. (1990) found that the variations of the $\mathrm{H} \alpha \lambda 6563$ and $\mathrm{H} \beta \lambda 4861$ emission lines lag behind those of the blue continuum by $7 \pm 3$ days.

\section{THE OBSERVATIONS}

The nucleus of NGC 5548 was observed at 60 different epochs in the low-resolution mode $\left(1000 \mathrm{~km} \mathrm{~s}^{-1}\right)$ and through the large apertures $\left(10^{\prime \prime} \times 20^{\prime \prime}\right)$ of the IUE (Boggess et al. $1978 a, b$; Faelker, Gordon, and Sandford 1987) spectrographs. Each epoch lasted about $4 \mathrm{hr}$ (exceptionally $8 \mathrm{hr}$ ), during which

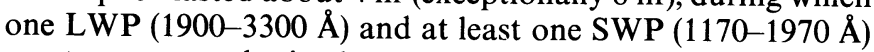
spectrum were obtained. In total, the data base contains 85 SWP spectra and 61 LWP spectra. Their sequential numbers 
TABLE 1

LOG OF IUE OBSERVATIONS

\begin{tabular}{|c|c|c|c|c|c|c|}
\hline Image $\mathrm{N}^{\circ}$ & $\begin{array}{l}\text { Date } \\
\text { U.T. }\end{array}$ & $\begin{array}{c}\text { Start Time } \\
\text { (Hour:Min:Sec) }\end{array}$ & $\begin{array}{c}\text { J.D. } \\
(-2,440,000)\end{array}$ & $\begin{array}{l}\text { Duration } \\
\text { (Seconds) }\end{array}$ & $\begin{array}{c}\text { FES } \\
\text { (Counts) }\end{array}$ & Notes \\
\hline LWP14644 & 14DEC88 & $14: 02: 06$ & 7510.08479 & 3900 & 53 & \\
\hline SWP35029 & 14DEC88 & $15: 20: 48$ & 7510.13944 & 5220 & 47 & \\
\hline SWP35070 & 18DEC88 & $13: 57: 29$ & 7514.08159 & 5400 & 53 & \\
\hline LWP14664 & 18DEC88 & $15: 35: 48$ & 7514.14986 & 3900 & 53 & \\
\hline SWP35097 & 22DEC88 & $10: 13: 03$ & 7517.92573 & 5400 & 52 & \\
\hline LWP14693 & 22DEC88 & $11: 51: 46$ & 7517.99428 & 3900 & 54 & \\
\hline SWP35138 & 26DEC88 & $14: 11: 05$ & 7522.09103 & 5400 & 51 & Cosmic ray \\
\hline LWP14721 & 26DEC88 & $15: 48: 08$ & 7522.15843 & 3600 & 54 & \\
\hline SWP35190 & 30DEC88 & $14: 22: 39$ & 7526.09906 & 5400 & 54 & \\
\hline LWP14744 & 30DEC88 & $16: 01: 25$ & 7526.16765 & 3000 & 56 & \\
\hline SWP35242 & 03JAN89 & $12: 18: 26$ & 7530.01280 & 6000 & 49 & \\
\hline LWP14763 & 03JAN89 & $14: 05: 03$ & 7530.08684 & 2400 & 50 & \\
\hline SWP35284 & 07JAN89 & $12: 03: 05$ & 7534.00214 & 5400 & 54 & \\
\hline LWP14784 & 07JAN89 & $13: 43: 20$ & 7534.07176 & 3900 & 56 & \\
\hline SWP35323 & 11JAN89 & $16: 04: 58$ & 7538.17012 & 5100 & 54 & \\
\hline SWP35324 & $11 \mathrm{JAN} 89$ & $18: 22: 16$ & 7538.26546 & 4800 & 56 & \\
\hline LWP14802 & 11JAN89 & $17: 43: 10$ & 7538.23831 & 3600 & 55 & \\
\hline SWP35325 & 11JAN89 & $20: 30: 59$ & 7538.35485 & 4800 & 62 & \\
\hline SWP35363 & $16 \mathrm{JAN} 89$ & $17: 08: 41$ & 7543.21436 & 3900 & 56 & \\
\hline LWP14848 & 16JAN89 & $18: 21: 42$ & 7543.26507 & 3000 & 57 & \\
\hline SWP35364 & $16 \mathrm{JAN} 89$ & $19: 20: 26$ & 7543.30586 & 3300 & 60 & \\
\hline SWP35383 & 19JAN89 & $12: 12: 23$ & 7546.00860 & 5400 & 54 & \\
\hline LWP14870 & 19JAN89 & $13: 52: 39$ & 7546.07823 & 3600 & 55 & \\
\hline SWP35393 & 23JAN89 & $07: 55: 58$ & 7549.83053 & 5400 & 55 & \\
\hline LWP14901 & 23JAN89 & $09: 33: 24$ & 7549.89819 & 3900 & 57 & \\
\hline SWP35416 & 26JAN89 & $15: 50: 35$ & 7553.16013 & 3600 & 55 & \\
\hline LWP14927 & 26JAN89 & $16: 58: 51$ & 7553.20753 & 2700 & 56 & \\
\hline SWP35461 & 30JAN89 & $15: 49: 26$ & 7557.15933 & 3600 & 53 & \\
\hline LWP14945 & 30JAN89 & $16: 58: 29$ & 7557.20728 & 2700 & 53 & \\
\hline SWP35462 & 30JAN89 & $17: 50: 57$ & 7557.24372 & 3600 & 56 & \\
\hline SWP35478 & 04FEB89 & $08: 31: 38$ & 7561.85530 & 5400 & 50 & \\
\hline LWP14964 & 04FEB89 & $10: 09: 34$ & 7561.92331 & 3000 & 50 & \\
\hline LWP14970 & 05FEB89 & 17:58:00 & 7563.24861 & 3900 & 57 & Few px sat. \\
\hline SWP35511 & 08FEB89 & $08: 54: 23$ & 7565.87110 & 4800 & 51 & Vignetting \\
\hline LWP14989 & 08FEB89 & $.10: 21: 18$ & 7565.93146 & 2700 & 51 & Vignetting \\
\hline SWP35538 & 12 FEB89 & $08: 16: 35$ & 7569.84485 & 6000 & 46 & \\
\hline LWP15024 & 12FEB89 & $10: 03: 38$ & 7569.91919 & 3600 & 48 & \\
\hline SWP35569 & 16 FEB89 & $05: 06: 58$ & 7573.71317 & 5400 & 44 & \\
\hline LWP15045 & 16FEB89 & $06: 57: 33$ & 7573.78997 & 2100 & 45 & \\
\hline LWP15067 & 20FEB89 & 09:06:09 & 7577.87927 & 3000 & 44 & \\
\hline SWP35595 & 20FEB89 & $10: 03: 43$ & 7577.91925 & 3240 & 44 & \\
\hline SWP35617 & 24FEB89 & $08: 46: 33$ & 7581.86566 & 5400 & 44 & \\
\hline LWP15087 & 24FEB89 & $10: 26: 38$ & 7581.93516 & 2100 & 45 & \\
\hline LWP15113 & 28FEB89 & $11: 45: 55$ & 7585.99022 & 3000 & 44 & \\
\hline SWP35636 & 28FEB89 & $12: 19: 21$ & 7586.01344 & 5400 & 46 & \\
\hline SWP35637 & 28FEB89 & $14: 34: 09$ & 7586.10705 & 1620 & 47 & \\
\hline SWP35676 & 04MAR89 & $11: 46: 48$ & 7589.99083 & 4800 & 47 & \\
\hline LWP15131 & 04MAR89 & $13: 15: 21$ & 7590.05233 & 2700 & 48 & \\
\hline SWP35677 & 04MAR89 & 14:08:11 & 7590.08902 & 2700 & 49 & \\
\hline SWP35712 & 08MAR89 & $12: 00: 48$ & 7594.00056 & 4800 & 46 & \\
\hline LWP15152 & 08MAR89 & $13: 30: 03$ & 7594.06253 & 2700 & 49 & \\
\hline
\end{tabular}

are listed in the observing log of Table 1 . The acquisition procedure was standard. Particular care was taken to center accurately the nucleus inside the aperture with the Fine Error Sensor (FES) star tracker. The exposure times were chosen such that the maximum allowable signal level is reached at the top of the $\operatorname{Ly} \alpha \lambda 1216$ and $\mathrm{Mg}$ II $\lambda 2798$ emission lines. Three
LWP spectra and one SWP spectrum turned out to have 1 pixel saturated each. Such a level of saturation is so small that it has no significant impact. A few hours prior to the observations of 1989 February 8, the spectrograph apertures had been closed. Closing the aperture has the effect of shifting the FES reference frame by up to $5^{\prime \prime}$ (Sonneborn et al. 1987). The posi- 
TABLE 1-Continued

\begin{tabular}{|c|c|c|c|c|c|c|}
\hline Image $N^{\circ}$ & $\begin{array}{l}\text { Date } \\
\text { U.T. }\end{array}$ & $\begin{array}{c}\text { Start Time } \\
\text { (Hour:Min:Sec) }\end{array}$ & $\begin{array}{c}\text { J.D. } \\
(-2,440,000)\end{array}$ & $\begin{array}{l}\text { Duration } \\
\text { (Seconds) }\end{array}$ & $\begin{array}{c}\text { FES } \\
\text { (Counts) }\end{array}$ & Notes \\
\hline SWP35713 & 08MAR89 & $14: 25: 47$ & 7594.10124 & 1800 & 49 & \\
\hline SWP35753 & 12MAR89 & $11: 57: 05$ & 7597.99797 & 4200 & 46 & \\
\hline LWP15174 & 12MAR89 & $13: 17: 11$ & 7598.05360 & 2700 & 51 & \\
\hline SWP35754 & 12MAR89 & $14: 09: 39$ & 7598.09003 & 2400 & 51 & \\
\hline SWP 35789 & 16MAR89 & $11: 55: 51$ & 7601.99712 & 4200 & 47 & \\
\hline LWP15202 & 16MAR89 & $13: 17: 10$ & 7602.05359 & 3000 & 50 & \\
\hline SWP35790 & 16MAR89 & $14: 16: 10$ & 7602.09456 & 3300 & 50 & \\
\hline SWP35823 & 20MAR89 & $12: 13: 43$ & 7606.00953 & 3900 & 49 & \\
\hline LWP15227 & 20MAR89 & $13: 30: 00$ & 7606.06250 & 3000 & 51 & \\
\hline SWP35824 & 20MAR89 & $14: 27: 57$ & 7606.10274 & 1440 & 53 & \\
\hline SWP 35849 & 24MAR89 & $09: 42: 53$ & 7609.90478 & 4200 & 48 & \\
\hline SWP35880 & 28MAR89 & $04: 26: 21$ & 7613.68497 & 5400 & 48 & \\
\hline LWP15254 & 28MAR89 & $06: 07: 30$ & 7613.75521 & 3600 & 50 & \\
\hline LWP15281 & 01APR89 & $01: 33: 55$ & 7617.56522 & 3600 & 57 & \\
\hline SWP35907 & 01APR89 & $02: 57: 28$ & 7617.62324 & 6000 & 49 & \\
\hline SWP35936 & 05APR89 & $02: 07: 17$ & 7621.58839 & 5400 & 53 & \\
\hline LWP15305 & 05APR89 & $03: 45: 35$ & 7621.65666 & 3900 & 54 & \\
\hline SWP35965 & 09APR89 & $02: 10: 20$ & 7625.59051 & 5400 & 53 & \\
\hline LWP15327 & 09APR89 & $03: 49: 46$ & 7625.65956 & 3900 & 56 & 1 px sat. \\
\hline SWP35996 & 13APR89 & $05: 45: 37$ & 7629.74001 & 4800 & 56 & \\
\hline LWP15350 & 13APR89 & $07: 16: 50$ & 7629.80336 & 3300 & 54 & \\
\hline SWP35997 & 13APR89 & $08: 17: 12$ & 7629.84528 & 2100 & 53 & \\
\hline SWP36018 & 17APR89 & $10: 13: 11$ & 7633.92582 & 3900 & 53 & \\
\hline LWP15361 & 17APR89 & $11: 29: 39$ & 7633.97892 & 3000 & 55 & \\
\hline SWP36019 & 17APR89 & $12: 25: 02$ & 7634.01738 & 1560 & 57 & \\
\hline SWP36055 & 21APR89 & $09: 59: 19$ & 7637.91619 & 3900 & 55 & \\
\hline LWP15385 & 21APR89 & $11: 15: 44$ & 7637.96926 & 3000 & 55 & \\
\hline SWP36056 & 21APR89 & $12: 17: 01$ & 7638.01182 & 2040 & 55 & \\
\hline SWP36089 & 25APR89 & $11: 32: 28$ & 7641.98088 & 3900 & 54 & \\
\hline LWP15406 & 25APR89 & $12: 45: 28$ & 7642.03157 & 3000 & 54 & \\
\hline SWP36159 & 29APR89 & $09: 45: 13$ & 7645.90640 & 3600 & 56 & \\
\hline LWP15410 & 29APR89 & $10: 56: 11$ & 7645.95568 & 2700 & 56 & \\
\hline SWP36160 & 29APR89 & $11: 50: 06$ & 7645.99312 & 3600 & 56 & \\
\hline SWP36184 & 03MAY89 & 08:09:39 & 7649.84003 & 3900 & 52 & \\
\hline LWP15443 & 03MAY89 & $09: 26: 41$ & 7649.89353 & 3000 & 55 & \\
\hline SWP36185 & 03MAY89 & $10: 26: 26$ & 7649.93502 & 1500 & 54 & \\
\hline SWP36210 & 07MAY89 & $08: 24: 11$ & 7653.85013 & 3900 & 54 & \\
\hline LWP15473 & 07MAY89 & $09: 37: 27$ & 7653.90101 & 3000 & 55 & \\
\hline SWP36240 & 11MAY89 & $00: 34: 22$ & 7657.52387 & 4200 & 57 & \\
\hline LWP15492 & 11MAY89 & $01: 53: 29$ & 7657.57881 & 3300 & 56 & \\
\hline SWP36264 & 15MAY89 & $00: 02: 20$ & 7661.50162 & 4500 & 53 & \\
\hline LWP15517 & 15MAY 89 & $01: 32: 48$ & 7661.56444 & 3600 & 51 & \\
\hline SWP36296 & 18MAY 89 & $23: 52: 43$ & 7665.49494 & 4800 & 52 & 1 px sat. \\
\hline LWP15542 & 19MAY89 & $01: 39: 25$ & 7665.56904 & 3600 & 53 & 1 px sat. \\
\hline SWP 36322 & 23MAY 89 & $00: 02: 38$ & 7669.50183 & 3600 & 61 & \\
\hline LWP15578 & 23MAY 89 & $01: 13: 45$ & 7669.55122 & 3600 & 51 & 1 px sat. \\
\hline SWP36323 & 23MAY89 & $02: 22: 23$ & 7669.59888 & 3000 & 49 & \\
\hline LWP15606 & 27MAY89 & $00: 11: 45$ & 7673.50816 & 3300 & 50 & \\
\hline SWP36345 & 27MAY 89 & $01: 13: 46$ & 7673.55123 & 4500 & 50 & \\
\hline SWP36369 & 31MAY89 & $00: 27: 43$ & 7677.51925 & 4500 & 50 & \\
\hline
\end{tabular}

tion of the guide star as measured by the FES, as well as the global shift of the wavelength scales on that day, show that the nucleus of NGC 5548 was offset by 4 " from the center of the aperture, along its small axis. The data therefore suffered from significant vignetting.

Immediately before (and some time after) each exposure, the optical brightness of NGC 5548 was recorded with the FES star tracker on board IUE. The FES is an image dissector which provides broad-band photometric flux measurements down to an rms accuracy of $\pm 0.07 \mathrm{mag}$ (Holm and Crabb 1979; Barylak, Wasatonic, and Imhoff 1984). The response curve of its S-20 photocathode extends from 3900 to $9000 \AA$, with a sharp maximum of sensitivity near $4600 \AA$ (Holm and Rice 1981). For an AGN-type spectrum, its effective wavelength is $\sim 5000 \AA$. In its "overlap" tracking mode, the equivalent aperture of the FES is $12^{\prime \prime} \times 12^{\prime \prime}$ squared. Hence, the NGC 5548 count rates include a significant contribution from the underlying stellar population. The FES is known to suffer from 
TABLE $1-$ Continued

\begin{tabular}{|c|c|c|c|c|c|c|}
\hline Image $\mathrm{N}^{\circ}$ & $\begin{array}{l}\text { Date } \\
\text { U.T. }\end{array}$ & $\begin{array}{l}\text { Start Time } \\
\text { (Hour:Min:Sec) }\end{array}$ & $\begin{array}{c}\text { J.D. } \\
(-2,440,000)\end{array}$ & $\begin{array}{l}\text { Duration } \\
\text { (Seconds) }\end{array}$ & $\begin{array}{c}\text { FES } \\
\text { (Counts) }\end{array}$ & Notes \\
\hline LWP15626 & 31MAY89 & $01: 51: 28$ & 7677.57741 & 3600 & 48 & \\
\hline SWP36385 & 03JUN89 & $05: 52: 41$ & 7680.74492 & 4500 & 51 & \\
\hline LWP15647 & 03JUN89 & $07: 26: 28$ & 7680.81005 & 3000 & 50 & \\
\hline SWP36386 & 03JUN89 & $08: 27: 14$ & 7680.85225 & 1440 & 50 & \\
\hline LWP15670 & 07JUN89 & $09: 17: 51$ & 7684.88740 & 3000 & 49 & \\
\hline SWP36411 & 07JUN89 & $10: 17: 43$ & 7684.92897 & 4500 & 49 & \\
\hline LWP15671 & 07 JUN89 & $11: 42: 09$ & 7684.98760 & 2100 & 50 & \\
\hline SWP36412 & 07JUN89 & $12: 28: 05$ & 7685.01950 & 1500 & 49 & \\
\hline SWP36446 & 11JUN89 & $05: 49: 08$ & 7688.74245 & 4500 & 47 & \\
\hline LWP15702 & 11JUN89 & $07: 13: 15$ & 7688.80087 & 3000 & 47 & \\
\hline SWP36447 & $11 \mathrm{JUN} 89$ & $08: 09: 41$ & 7688.84006 & 2400 & 47 & \\
\hline SWP36509 & 15JUN89 & $05: 49: 37$ & 7692.74279 & 4500 & 47 & \\
\hline LWP15729 & 15JUN89 & $07: 13: 55$ & 7692.80133 & 3000 & 48 & \\
\hline SWP36510 & 15JUN89 & $08: 13: 54$ & 7692.84299 & 2400 & 46 & \\
\hline SWP36551 & 19JUN89 & $06: 09: 37$ & 7696.75668 & 4500 & 48 & \\
\hline LWP15750 & 19JUN89 & $07: 33: 19$ & 7696.81480 & 3000 & 48 & \\
\hline SWP36567 & 23JUN89 & $05: 45: 16$ & 7700.73977 & 4500 & 46 & \\
\hline LWP15773 & 23JUN89 & $07: 09: 40$ & 7700.79838 & 3000 & 47 & \\
\hline SWP36568 & 23JUN89 & $08: 07: 33$ & 7700.83858 & 2580 & 48 & \\
\hline SWP 36580 & $27 J U N 89$ & $22: 18: 48$ & 7705.42972 & 4800 & 49 & \\
\hline LWP15805 & 27JUN89 & $23: 47: 17$ & 7705.49117 & 3600 & 48 & \\
\hline SWP 36595 & 01JUL89 & $20: 09: 45$ & 7709.34010 & 4800 & 48 & \\
\hline LWP15822 & 01JUL89 & $21: 40: 02$ & 7709.40280 & 3600 & 50 & \\
\hline SWP36616 & 05JUL89 & $20: 02: 07$ & 7713.33480 & 4800 & 49 & \\
\hline LWP15857 & 05JUL89 & $21: 30: 45$ & 7713.39635 & 3600 & 50 & \\
\hline SWP36648 & 09JUL89 & $19: 44: 42$ & 7717.32271 & 4200 & 53 & \\
\hline LWP15889 & 09JUL89 & $21: 05: 23$ & 7717.37874 & 3300 & 53 & \\
\hline SWP 36675 & 13JUL89 & $20: 02: 18$ & 7721.33493 & 4800 & 49 & \\
\hline LWP15912 & 13JUL89 & $21: 40: 48$ & 7721.40333 & 3600 & 49 & \\
\hline SWP 36691 & 17 JUL89 & $20: 04: 52$ & 7725.33671 & 4200 & 44 & \\
\hline LWP15948 & 17 JUL 89 & $21: 23: 55$ & 7725.39161 & 3300 & 48 & \\
\hline SWP36692 & 17 JUL89 & $22: 27: 51$ & 7725.43601 & 1800 & 45 & \\
\hline SWP36715 & 22JUL89 & 04:04:51 & 7729.67003 & 4500 & 46 & \\
\hline LWP15976 & 22JUL 89 & $05: 29: 11$ & 7729.72860 & 3000 & 48 & \\
\hline SWP36716 & 22JUL 89 & $06: 25: 40$ & 7729.76782 & 1500 & 49 & \\
\hline SWP36739 & 26JUL89 & $04: 19: 38$ & 7733.68030 & 4500 & 45 & \\
\hline LWP15989 & 26JUL89 & $05: 40: 13$ & 7733.73626 & 3000 & 46 & \\
\hline SWP 36740 & 26JUL89 & $06: 36: 04$ & 7733.77505 & 1200 & 46 & \\
\hline SWP36758 & 30JUL 89 & $03: 53: 47$ & 7737.66235 & 4500 & 45 & \\
\hline LWP 16019 & 30 JUL 89 & $05: 18: 05$ & 7737.72089 & 3000 & 45 & \\
\hline SWP 36759 & 30JUL89 & $06: 13: 34$ & 7737.75942 & 2100 & 45 & \\
\hline SWP 36783 & 03AUG89 & $01: 58: 38$ & 7741.58238 & 5700 & 43 & \\
\hline LWP 16055 & 03AUG89 & $03: 44: 06$ & 7741.65563 & 3900 & 41 & \\
\hline SWP36805 & 07AUG89 & $01: 21: 53$ & 7745.55686 & 5100 & 43 & \\
\hline LWP16083 & 07AUG89 & 02:53:04 & 7745.62019 & 2580 & 44 & \\
\hline
\end{tabular}

a gradual loss of sensitivity (Barylak, Wasatonic, and Imhoff 1984). However, the decrease in efficiency over an 8 month period is so small that it may be neglected. The FES counts are listed in Table 1.

\section{DATA REDUCTION AND ANALYSIS}

In order to test the robustness of our results, the ultraviolet spectra were reduced independently using two different methods. Similarly, the continuum and line fluxes were independently measured by different people using different procedures. We now describe briefly each of these methods.

\section{a) IUESIPS Data Reduction and Flux Summation}

In the first method, the spectra have been reduced with the standard IUE Spectral Image Processing System (IUESIPS), which is routinely used by the $I U E$ project to process guest observers' data (Turnrose and Thompson 1984; Harris and Sonneborn 1987). After the pixel-to-pixel photometric calibration ("ITF correction"), a " gross" spectrum is extracted by summing at each wavelength step all the flux which falls into an 18 pixel wide (19".4) slit. A smoothed background measured on each side of the data strip is then subtracted from the gross 
tral extraction method was also applied to the data. The inputs to the procedure were the two-dimensional, geometrically and photometrically corrected ELBL files as processed by the standard IUESIPS software. Prior to the extraction, the images were cleaned of cosmic-ray hits and other blemishes. The background regions on either side of the spectrum were cleaned by calculating the flux percentiles within smaller regions, and replacing the fluxes which fell outside the upper or lower 2 percentiles by the median values. Blemishes in the source region were removed interactively by interpolation over the affected pixels.

The optimal extraction method used was a modified version of the Gaussian extraction (GEX) technique. Details concerning the standard GEX technique are given in the discussion by Urry and Reichert (1988). Briefly, the two-dimensional data are summed over bins in wavelength, and each binned crossdispersive profile is fitted to a Gaussian point-spread function (PSF) plus a linear background. Two passes through the data are made. In the first pass, large wavelength bins are used, and the background and Gaussian parameters are all allowed to vary freely. In the second pass the data are summed over smaller wavelength bins, and only the normalizations of the Gaussians are allowed to vary. All other parameters are determined from the larger bin values, either by fitting to a quadratic dependence on wavelength or by linear interpolation. The net spectral fluxes are given by the integrals under the Gaussian fits to the smaller bins.

The version of GEX used in this work incorporates several improvements over the standard GEX, taking advantage of a better knowledge of the IUE camera's PSFs and the varying $\mathrm{S} / \mathrm{N}$ in the NGC 5548 spectra. Details concerning the necessity of the improvements and their implementation are given by Reichert and Kriss (1990). The modifications are as follows: (1) Cassatella, Barbero, and Benvenuti (1985, hereafter CBB) have shown that the PSF in the SWP camera is better fitted by a skewed Gaussian than by a symmetrical Gaussian. The procedure was therefore modified to fit skewed Gaussians to the cross-dispersive SWP profiles, with the skew parameters fixed according to the curve determined by CBB. (2) The procedure was modified to allow the cross-dispersive profiles to be summed over bins of variable width, so that comparatively wide bins could be used for the continuum regions and regions of lower camera sensitivity, and narrower bins used for the emission lines and regions of higher camera sensitivity. (3) The Gaussian widths for the second pass through the data were determined by higher order polynomial fits to the first-pass values. Fourth-order polynomials were fitted to the SWP widths, while fifth-order polynomials were used for the LWP widths. (4) Finally, the conversion from net to absolute spectral fluxes was calibrated using fits to bright standard stars.

In the absence of a true noise model for $I U E$ data, it can be difficult to derive true uncertainties for the extracted fluxes. However, assuming that the fitted Gaussians (or skewed Gaussians) are good representations of the cross-dispersive profiles, and barring systematic effects, then the deviations between the fitted models and the data should give a measure of the uncertainties. We have therefore adopted the deviation between the fitted model and the data as an estimate of the statistical uncertainty associated with each flux. The advantage of this method is that it allows independent estimates of the flux uncertainties to be determined for each flux, and can be used for the majority of epochs when only one spectrum per camera was obtained.
As a check on this method of estimating uncertainties, we have also estimated uncertainties by averaging nearconcurrent spectra on a pixel-by-pixel basis, and computing the residuals about these averages. By repeating this process for a number of spectral pairs, we derived distributions of residuals at various wavelengths for each camera. The widths of the distributions are measures of the uncertainties. In every case, the uncertainties derived from the GEX fits to the crossdispersive profiles closely matched the widths of the distributions of residuals, supporting our use of the GEX uncertainties. Further justifications of the GEX uncertainties are discussed by Reichert and Kriss (1990).

We note that the GEX uncertainties should be considered purely statistical uncertainties, and as such probably do not include systematic uncertainties such as those due to fixed pattern noise, etc. (The residuals from the spectral pairs will also not include systematic uncertainties.) It is well established that $I U E$ data are subject to systematic uncertainties (see, e.g., Bohlin 1988), and this fact probably accounts for the high values of reduced $\chi^{2}$ derived from the fits to continuum and emission-line regions (see discussion below). Based on the results of these fits, we deduce that our estimated uncertainties represent approximately half of the combined statistical plus systematic uncertainties.

Emission-line and continuum fluxes in the GEX-extracted spectra are derived from multicomponent, least-squares fits to the data. Full details of the fitting procedure will be described in Reichert and Kriss (1990). Here we give a brief summary of the major steps.

For the SWP spectra, parameters in the least-squares fits include a power law of the form

$$
F_{\lambda}=F_{0}\left(\frac{\lambda}{1000}\right)^{-\beta}
$$

plus 14 Gaussian components: two for $\mathrm{Ly} \alpha$, five for C IV $\lambda 1549$, two for C III] $\lambda 1909$, one each for $N$ v $\lambda 1240$, O I $\lambda 1304$, $\mathrm{Si}$ IV $+\mathrm{O}$ IV] $\lambda 1402$, and He II $\lambda 1640+\mathrm{O}$ III] $\lambda 1663$, and one in optical depth for the $N$ v $\lambda 1240$ absorption feature. Each Gaussian component has its total flux, central wavelength, and its dispersion as free parameters. No physical significance is attached to any of these components. They merely serve as a convenient description of the data which permit accurate fluxes with suitable error bars to be determined.

Fits to the SWP spectra are done in a stepwise fashion that decouples parameters that are not closely linked. Starting from a fit to an average spectrum, initial guesses for the line and continuum fluxes are made by scaling this fit by previously determined quick-look answers for the continuum flux at 1337 $\AA$ and the C IV flux. The continuum is then fitted in the line-free windows $1317-1357 \AA$ plus $1720-1858 \AA$. With the continuum fixed, the Si IV + O IV blend, the $\mathrm{C}$ III] line, and the $\mathrm{C}$ IV and $\mathrm{He}$ II $+\mathrm{O}$ III] region are all fitted individually. Once initial fits have been made to each of these independent line complexes, the spectral range from 1350 to $1962 \AA$ (observed wavelengths) is fitted with all line and continuum parameters free to vary. A $7 \AA$ region surrounding the camera feature at $\lambda_{\text {obs }}=1666 \AA$ and a $9 \AA$ region around the reseau at $\lambda_{\text {obs }}=1799 \AA$ are excluded from the final fit.

Lines in the $\mathrm{Ly} \alpha$ region are fitted independently with the continuum fixed according to the fits redward of $1337 \AA$, since there is no good continuum shortward of Ly $\alpha$. An initial fit is performed with the width of broad Ly $\alpha$ and $\mathrm{N} v$ fixed. This 
yields better consistency in the final answers. After this iteration establishes the line fluxes and centers, a final fit is done in which all line components are allowed to vary freely.

Fits to the LWP spectra are complicated by the pseudocontinuum of $\mathrm{Fe}$ II emission, which is present throughout the LWP spectral range (see $\S$ III $a$ ). To avoid the complications of modeling the entire Fe II spectrum, only a small region around $\mathrm{Mg}$ II from 2540 to $3050 \AA$ is fitted. The window from $\lambda_{\text {obs }}=$ $3035 \AA$ to $\lambda_{\text {obs }}=3075 \AA$ is excluded to avoid a reseau. Strong blends of $\mathrm{Fe}$ II emission are modeled with broad Gaussians centered at 2595,2724 , and $2947 \AA$. The underlying pseudocontinuum of weaker $\mathrm{Fe}$ II lines and Balmer continuous emission is fitted with a power law. Comparing an average of all LWP spectrum to a model of $\mathrm{Fe}$ II emission (Netzer and Wills 1983) shows that the 2645-2695 $\AA$ wavelength interval represents a local minimum in the $\mathrm{Fe}$ II emission. It was therefore chosen to sample the continuum in the LWP. A single Gaussian describes the profile of $\mathrm{Mg}$ II $\lambda 2798$ well if an additional Gaussian is included to account for the bright $\mathrm{Fe}$ II feature at $2838 \AA$ in the models of Netzer and Wills (1983).

In fitting an individual LWP spectrum, the Fe II line fluxes are fixed, and the continuum parameters are adjusted using a wavelength range which avoids the $\mathrm{Mg}$ II line. Next, the continuum is fixed, and only the line fluxes are permitted to vary. In the final iteration all parameters are permitted to vary freely.

Error bars for the fitted parameters in both the SWP and the LWP spectra are calculated in a semiempirical way. Under the assumption that each parameter is a single "interesting" parameter (see Avni 1976), the $1 \sigma$ error $\sigma_{i}$ on a parameter $a_{i}$ is obtained from the error matrix of the fit, $\epsilon_{i j}$, scaled by the reduced chi-square, $\chi_{v}^{2}$ :

$$
\sigma_{i}^{2}=\chi_{v}^{2} \epsilon_{i i} .
$$

For observed quantities of interest which are functions of more than one parameter, e.g., the total flux of $C_{\text {IV }}$, the error bar is calculated using

$$
\sigma_{\mathrm{CIV} \mathrm{tot}}^{2}=\sum_{i} \sigma_{i}^{2}+\sum_{i \neq j} \sigma_{i j}^{2},
$$

where the cross-variance $\sigma_{i j}^{2}$ is the error matrix element $\epsilon_{i j}$ scaled by $\chi_{v}^{2}$. Typically, $\chi_{v}^{2}$ for the final fits ranged from 3 to 5 , indicating that the statistical uncertainties for the individual fluxes (derived from the deviations about the cross-dispersive profiles in the Gaussian extractions) probably underestimate the combined uncertainties by a factor of $\sim 2$.

We tested the appropriateness of the errors in the line and continuum fluxes by examining the scatter in the results for multiple observations. For the SWP we use pairs of spectra that were obtained during the same IUE shift. For the LWP we used adjacent pairs of spectra, since only one pair of LWP exposures were taken on the same shift. Under the assumption that there is no variability on such short time scales, the differences in line or continuum flux for the spectral pairs, divided by the estimated errors summed in quadrature, should show a Gaussian distribution with zero mean and unit dispersion if the error bars are appropriate and if there are no external sources of error. The distributions of the scaled differences for the fluxes have dispersions of $\sim 2$ for $\mathrm{C}$ IV and $\mathrm{Si}$ IV, $\sim 5$ for Ly $\alpha, \mathrm{N}$ v, He II, C III], and $F_{1813}$, and $\sim 7$ for $F_{1337}$. Thus, while the calculated errors are probably accurate measures of the statistical errors internal to a given spectrum, they do not assess the true external errors for the repeatability of IUE spectra. To account for these additional external errors, the tabulated errors for the final results were therefore scaled up by a factor equal to the dispersions measured for the scaled difference distributions of the spectral pairs.

\section{c) Comparison of Results}

In what follows, we shall refer to the IUESIPS data extraction plus flux summation method as SIPS, whereas the Gaussian extraction plus line and continuum fitting process will be called GEX, for simplicity.

The continuum flux at $1350 \AA$ (SIPS), $1337 \AA$ (GEX) $1840 \AA$ (SIPS), and $1813 \AA$ (GEX), the SWP frequency spectral index $\alpha$ $\left(F_{v} \propto v^{-\alpha}\right)$, as well as the $\operatorname{Ly} \alpha \lambda 1216+\mathrm{N} v \lambda 1240$ blend and $C$ IV $\lambda 1549$ line fluxes measured both with SIPS and GEX, are listed in Table 2 . We caution that the values of $\alpha$ listed slightly overestimate the true spectral index of the continuum, since no attempt has been made to remove the steep pseudocontinuum created by the many blended low-contrast $\mathrm{Fe}$ II lines and Balmer continuum emission (Wills, Netzer, and Wills 1985). In Table 3 we give the SIPS and GEX fluxes of $\mathrm{Si}$ IV $+\mathrm{O}$ iv] $\lambda 1402$, He II $\lambda 1640+\mathrm{O}$ III] $\lambda 1663, \mathrm{C}$ III] $\lambda 1909$, and N v $\lambda 1240$ (GEX only). Table 4 contains the $2670 \AA$ continuum and Mg II $\lambda 2798$ line SIPS and GEX fluxes. All fluxes are given as a function of the Julian Date and time at the start of the corresponding exposure.

Knowing the width of the IUE point-spread function and its wavelength dependence (CBB), it is possible to estimate the fraction of the flux which actually entered the spectrographs on 1989 February 8 and correct for the effect of vignetting. The correction factors which have been applied to each of the line and continuum fluxes are given in the footnotes to the tables. Because of their systematic uncertainty, however, the data of 1989 February 8 have not been included in the analysis of $\$$ IV and V. We checked, however, that their inclusion does not affect any of the results.

For time series analysis it is convenient to have only one flux measurement per epoch. The results obtained during the same observing shift were therefore averaged. In the case of the data reduced with SIPS, the averaging was performed after extraction of the individual line and continuum fluxes by computing their weighted mean. For the GEX data, the spectra obtained on the same day are first averaged using a weighted mean, and the fitting procedure is performed again on the mean spectra to measure the line and continuum fluxes and their associated errors. In the remainder of this paper, we shall be concerned with the averaged results only.

To compare the results obtained with SIPS and GEX, linear regression analysis was performed between both sets of measurements. The regression techniques used take into account the errors both on the dependent (GEX) and the independent (SIPS) variable simultaneously (York 1966). The results are given in Table 5, where we list the coefficients of the best-fit regression line, the correlation coefficient, the reduced chisquare, and the probability of exceeding $\chi_{v}^{2}$ by chance. In Figure 2 the GEX fluxes are plotted as a function of their SIPS counterpart for $F_{1337}$ and $F_{1350}, \mathrm{C}$ IV $\lambda 1549$, and $\mathrm{He}$ II $\lambda 1640+\mathrm{O}$ III] $\lambda 1663$. The agreement between the two data sets is excellent. The correlation coefficients $r$ and reduced chisquares are always highly significant. For the continuum fluxes, the $\chi_{v}^{2}$ values are significantly smaller than 0.5 . This is partly because the two methods are not statistically independent, nearly identical quantities being derived from the same data. In addition, the errors which enter into the calculation of $\chi_{v}^{2}$ include both internal statistical errors and external system- 
TABLE 2

SWP OBSERVATIONS

\begin{tabular}{|c|c|c|c|c|c|c|c|c|c|}
\hline$(-2,440,000)$ & $\begin{array}{l}F_{1350} \\
\text { SIPS }\end{array}$ & $\begin{array}{c}F_{1337} \\
\text { GEX } \\
\left(10^{-14} \mathrm{erg} \mathrm{s}^{-}\right.\end{array}$ & $\begin{array}{c}F_{1840} \\
\text { SIPS } \\
\left.{ }^{1} \mathrm{~cm}^{-2} \mathrm{~A}^{-1}\right)\end{array}$ & $\begin{array}{l}F_{1813} \\
\text { GEX }\end{array}$ & $\begin{array}{c}\alpha_{\nu} \\
\operatorname{GEX}\end{array}$ & $\begin{array}{l}\text { Ly } \alpha \lambda 121 \\
\text { SIPS }\end{array}$ & $\begin{array}{c}+N V \lambda 1240 \\
\text { GEX } \\
\left(10^{-14} \text { erg s }\right.\end{array}$ & $\begin{array}{l}\text { CIV } \lambda \\
\text { SIPS } \\
\mathrm{cm}^{-2} \text { ) }\end{array}$ & $\begin{array}{l}1549 \\
\text { GEX }\end{array}$ \\
\hline 7510.139 & $3.71 \pm 0.26$ & $3.63 \pm 0.12$ & $2.95 \pm 0.18$ & $3.02 \pm 0.08$ & $1.40 \pm 0.11$ & $456 \pm 17$ & $654 \pm 41$ & $638 \pm 23$ & $613 \pm 31$ \\
\hline 7514.082 & & $.00 \pm 0.15$ & $3.04 \pm 0.18$ & & & & & $717 \pm 23$ & $684 \pm 42$ \\
\hline 7517.926 & $4.21 \pm 0.26$ & $4.23 \pm 0.13$ & $3.19 \pm 0.18$ & $3.23 \pm 0.07$ & $1.12 \pm 0.10$ & $504 \pm 17$ & $670 \pm 50$ & $694 \pm 23$ & $668 \pm 25$ \\
\hline 7522.091 & $4.10 \pm 0.26$ & $4.78 \pm 0.16$ & $3.54 \pm 0.18$ & $3.70 \pm 0.09$ & $1.16 \pm 0.11$ & $530 \pm 17$ & $730 \pm 59$ & $740 \pm 23$ & $727 \pm 30$ \\
\hline 7526.099 & $5.10 \pm 0.26$ & $5.16 \pm 0.18$ & $3.58 \pm 0.18$ & $3.59 \pm 0.10$ & $0.82 \pm 0.12$ & $555 \pm 17$ & $663 \pm 57$ & $691 \pm 23$ & $673 \pm 35$ \\
\hline 7530.013 & $5.32 \pm 0.26$ & $5.18 \pm 0$ & $3.77 \pm 0$ & $76 \pm 0.09$ & $.94 \pm 0.10$ & $553 \pm 17$ & $769 \pm 60$ & $77 \pm 23$ & $62 \pm 24$ \\
\hline & & & $3.54 \pm 0.18$ & $3.64 \pm 0.09$ & $0.97 \pm 0.10$ & & $682 \pm 63$ & $643 \pm 23$ & $636 \pm 28$ \\
\hline 7538.170 & $6.14 \pm 0.26$ & $6.07 \pm 0.17$ & $4.27 \pm 0.18$ & $4.27 \pm 0.09$ & $0.85 \pm 0.09$ & $601 \pm 17$ & $838 \pm 70$ & $701 \pm 23$ & $660 \pm 25$ \\
\hline 7538.265 & $5.89 \pm 0.26$ & $6.05 \pm 0.17$ & $4.46 \pm 0.18$ & & $0.94 \pm 0.09$ & $601 \pm 17$ & $844 \pm 64$ & $727 \pm 23$ & $689 \pm 25$ \\
\hline 7538.355 & $5.89 \pm 0.26$ & $6.07 \pm 0$ & $4.71 \pm 0.18$ & $4.37 \pm 0.09$ & $0.92 \pm 0$ & \pm 17 & $932 \pm 82$ & $705 \pm 23$ & $735 \pm 28$ \\
\hline 7543.214 & $6.23 \pm 0.26$ & $6.38 \pm 0.21$ & $4.60 \pm 0.18$ & $4.53 \pm 0.11$ & $0.87 \pm 0.11$ & $619 \pm 17$ & $943 \pm 64$ & $765 \pm 23$ & $760 \pm 44$ \\
\hline 7543.306 & & $6.20 \pm 0$ & $4.47 \pm 0$ & & & & $884 \pm 67$ & $760 \pm 23$ & $710 \pm 38$ \\
\hline 7546.009 & $6.28=$ & $6.14 \pm 0.17$ & $4.52 \pm 0.18$ & $4.51 \pm 0.10$ & $0.99 \pm 0$ & & $932 \pm 61$ & $786 \pm 23$ & $765 \pm 35$ \\
\hline 7549.831 & 0.26 & $5.24 \pm 0.16$ & $3.83 \pm 0$ & 0.09 & $1.08 \pm$ & & & $796 \pm 23$ & $774 \pm 22$ \\
\hline & & $5.11 \pm 0.19$ & $3.80 \pm 0.18$ & $3.90 \pm 0.11$ & $1.11 \pm 0.12$ & & & $892 \pm 23$ & $863 \pm 50$ \\
\hline 7557.159 & $4.76 \pm 0.26$ & & $3.40 \pm 0.18$ & & & & & $795 \pm 23$ & $807 \pm 27$ \\
\hline 7557.244 & 0.26 & $4.80 \pm c$ & $3.45 \pm$ & & & & & $785 \pm 23$ & $763 \pm 33$ \\
\hline 7561.855 & $3.55 \pm 0.26$ & $26+0$ & $2.80 \pm 0.18$ & $2.75 \pm 0$ & 13 & & $720 \pm 51$ & $715 \pm 23$ & $736 \pm 39$ \\
\hline $7565.871^{a}$ & $2.99 \pm 0.26$ & $2.97 \pm 0.14$ & $2.90 \pm 0.18$ & $2.71 \pm 0.07$ & $1.41 \pm 0.16$ & \pm 17 & $812 \pm 50$ & $656 \pm 23$ & $633 \pm 24$ \\
\hline 7569.845 & $3.28 \pm$ & $3.22 \pm 0$ & $2.56 \pm$ & .08 & 13 & & \pm 50 . & $654 \pm 23$ & $672 \pm 53$ \\
\hline 7573.713 & $3.00 \pm 0.26$ & $3.15 \pm 0.13$ & $2.25 \pm 0.18$ & $2.33 \pm 0.07$ & $1.02 \pm 0.13$ & $475 \pm 17$ & $571 \pm 50$ & $622 \pm 23$ & $619 \pm 23$ \\
\hline 7577.919 & $.88 \pm 0.26$ & $.53 \pm 0.16$ & $2.29 \pm$ & & & & & $656 \pm 23$ & $600 \pm 33$ \\
\hline 7581.866 & & & $2.11 \pm 0$. & & & & & $47 \pm 23$ & $541 \pm 20$ \\
\hline & & & & & & & & $582 \pm 23$ & $588 \pm 18$ \\
\hline 7586.107 & $2.83 \pm 0.39$ & $2.66 \pm 0.21$ & $2.10 \pm 0.27$ & $2.13 \pm 0.13$ & $1.28 \pm 0.25$ & & $497 \pm 47$ & $583 \pm 35$ & $510 \pm 47$ \\
\hline 7589.991 & $3.19 \pm 0.26$ & $3.10 \pm 0.14$ & $2.40 \pm 0.18$ & $2.46 \pm 0.08$ & $1.24 \pm 0.14$ & & $505 \pm 29$ & $596 \pm 23$ & $602 \pm 21$ \\
\hline & & & & & & & & $99 \pm 23$. & \pm 11 \\
\hline & & & & & & & & $85 \pm 23$. & $594 \pm 13$ \\
\hline 7594.101 & 0.26 & $2.75 \pm 0.21$ & $2.11 \pm 0.1$ & $2.22 \pm 0.13$ & .24 & \pm 17 & $487 \pm 56$ & $644 \pm 23$ & $584 \pm 29$ \\
\hline 7597.998 & $4.18 \pm 0.26$ & $4.04 \pm 0.18$ & $2.90 \pm 0.18$ & $3.02 \pm 0.10$ & $1.04 \pm 0.14$ & & & $594 \pm 23$ & $557 \pm 10$ \\
\hline & & & & & & & & & \\
\hline & & & & & & & & $93 \pm 23$ & $556 \pm 18$ \\
\hline 7602.095 & $.20 \pm 0.26$ & $4.08 \pm 0.16$ & $3.23 \pm 0$ & 10 & 13 & \pm 17 & $633 \pm 53$ & $625 \pm 23$ & $619 \pm 27$ \\
\hline 7606.010 & $4.46 \pm 0.26$ & $4.43 \pm 0.16$ & $3.43 \pm 0.18$ & $3.47 \pm 0.10$ & $1.19 \pm 0.12$ & $453 \pm 17$ & $615 \pm 54$ & $612 \pm 23$ & $616 \pm 24$ \\
\hline 7606.103 & $3.90 \pm 0.39$ & $3.78 \pm 0.23$ & $3.23 \pm 0.27$ & $3.07 \pm 0.14$ & $1.32 \pm 0.20$ & $450 \pm 26$ & $699 \pm 60$ & $642 \pm 35$ & $664 \pm 32$ \\
\hline 7609.905 & & $4.76 \pm 0.62$ & & 11 & & & & $580 \pm 23$ & $632 \pm 21$ \\
\hline 7613.685 & $5.29 \pm 0.26$ & $5.42 \pm 0.15$ & $3.97 \pm 0.18$ & $3.98 \pm 0.08$ & & $6 \pm 17$ & $745 \pm 59$ & $684 \pm 23$ & $669 \pm 20$ \\
\hline 7617.623 & $5.30 \pm 0.26$ & $5.31 \pm 0.14$ & $3.75 \pm 0.18$ & $3.80 \pm 0.08$ & $0.90 \pm 0.09$ & $503 \pm 17$ & $709 \pm 45$ & $668 \pm 23$ & $700 \pm 11$ \\
\hline 7621.588 & $7.09 \pm 0.26$ & $7.17 \pm 0.20$ & $4.70 \pm 0.18$ & $4.89 \pm 0.10$ & $0.74 \pm 0.09$ & $590 \pm 17$ & & $745 \pm 23$ & $748 \pm 16$ \\
\hline 7625.591 & $6.46 \pm 0.26$ & $6.86 \pm 0.16$ & $4.63 \pm 0.18$ & $4.76 \pm 0.08$ & $0.80 \pm 0.08$ & & & $769 \pm 23$ & $772 \pm 12$ \\
\hline 7629.740 & $6.91 \pm 0.26$ & $6.91 \pm 0.18$ & $4.73 \pm 0.18$ & $4.83 \pm 0.10$ & $0.83 \pm 0.09$ & $603 \pm 17$ & $874 \pm 72$ & $776 \pm 23$ & $759 \pm 22$ \\
\hline 7629.845 & $6.63 \pm 0.26$ & $6.41 \pm 0.32$ & $4.61 \pm 0.18$ & $4.68 \pm 0.18$ & $0.96 \pm 0.16$ & $591 \pm 17$ & $989 \pm 73$ & $738 \pm 23$ & $716 \pm 28$ \\
\hline 7633.926 & $6.43 \pm 0.26$ & $6.50 \pm 0.19$ & $4.80 \pm 0.18$ & $4.73 \pm 0.10$ & $0.96 \pm 0.10$ & $672 \pm 17$ & $997 \pm 76$ & $755 \pm 23$ & $741 \pm 19$ \\
\hline 7634.017 & $6.11 \pm 0.39$ & $6.21 \pm 0.29$ & $4.10 \pm 0.27$ & $4.33 \pm 0.16$ & $0.82 \pm 0.16$ & $592 \pm 26$ & $706 \pm 84$ & $771 \pm 35$ & $727 \pm 35$ \\
\hline 7637.916 & $6.28 \pm 0.26$ & $6.37 \pm 0.21$ & $4.42 \pm 0.18$ & $4.57 \pm 0.12$ & $0.91 \pm 0.11$ & $707 \pm 17$ & $955 \pm 77$ & $789 \pm 23$ & $778 \pm 43$ \\
\hline
\end{tabular}

atic uncertainties, whereas only the internal errors are relevant when comparing the GEX and SIPS results.

Apart from the He II $\lambda 1640$ and Ly $\alpha \lambda 1216$ lines, the slope of the best-fit regression lines never differs from unity by more than $2 \sigma$, while the intercepts are null to within the error bars. The GEX fluxes for Ly $\alpha \lambda 1216$ are systematically larger by $\sim 50 \%$ than the SIPS values. This is a direct consequence of the fitting procedure which "restores" part of the blue wing occulted under the geocoronal emission feature. The GEX measurements of the $\mathrm{He}$ II $\lambda 1640+\mathrm{O}$ III] $\lambda 1663$ blend are almost twice as large as their SIPS counterpart. This difference appears to be primarily due to the way the fitting procedure deblends the He II $\lambda 1640+\mathrm{O}$ III $] \lambda 1663$ feature from the broad red wing of the $\mathrm{C}$ IV $\lambda 1549$ line. The SIPS integration interval is chosen to be symmetrical about $1640 \AA$, with a width determined from the local minimum of the flux in the interval 
TABLE 2-Continued

\begin{tabular}{|c|c|c|c|c|c|c|c|c|c|}
\hline$(-2,440,000)$ & $\begin{array}{l}F_{1350} \\
\text { SIPS }\end{array}$ & $\begin{array}{c}F_{1337} \\
\text { GEX } \\
\left(10^{-14} \mathrm{erg} \mathrm{s}^{-}\right.\end{array}$ & $\begin{array}{c}F_{1840} \\
\text { SIPS } \\
\left.{ }^{1} \mathrm{~cm}^{-2} \AA^{-1}\right)\end{array}$ & $\begin{array}{l}F_{1813} \\
\text { GEX }\end{array}$ & $\begin{array}{c}\alpha_{\nu} \\
\text { GEX }\end{array}$ & $\begin{array}{l}\text { Ly } \alpha \lambda 1216 \\
\text { SIPS }\end{array}$ & $\begin{array}{c}+\mathrm{NV} \lambda 1240 \\
\text { GEX } \\
\left(10^{-14} \mathrm{erg} \mathrm{s}^{-}\right.\end{array}$ & $\begin{array}{l}\text { CIV } \lambda \\
\text { SIPS } \\
\left.\mathrm{m}^{-2}\right)\end{array}$ & $\begin{array}{l}1549 \\
\text { GEX }\end{array}$ \\
\hline 7638.012 & $5.94 \pm 0.26$ & $5.99 \pm 0.34$ & $4.27 \pm 0.18$ & $4.41 \pm 0.10$ & $1.00 \pm 0.14$ & $609 \pm 17$ & $792 \pm 70$ & $757 \pm 23$ & $697 \pm 49$ \\
\hline 7641.981 & $6.56 \pm 0.26$ & $6.70 \pm 0.20$ & $5.02 \pm 0.18$ & $5.01 \pm 0.11$ & $1.05 \pm 0.10$ & $728 \pm 17$ & $1033 \pm 79$ & $834 \pm 23$ & $767 \pm 33$ \\
\hline 7645.906 & $6.46 \pm 0.26$ & $6.53 \pm 0.21$ & $4.26 \pm 0.18$ & $4.61 \pm 0.11$ & $0.86 \pm 0.11$ & $757 \pm 17$ & $1069 \pm 80$ & $907 \pm 23$ & $832 \pm 41$ \\
\hline 7645.993 & $6.24 \pm 0.26$ & $6.60 \pm 0.22$ & $4.50 \pm 0.18$ & $4.53 \pm 0.12$ & $0.77 \pm 0.11$ & $725 \pm 17$ & $1073 \pm 81$ & $849 \pm 23$ & $846 \pm 44$ \\
\hline 7649.840 & $5.82 \pm 0.26$ & $5.88 \pm 0.21$ & $4.33 \pm 0.18$ & $4.35 \pm 0.12$ & $1.02 \pm 0.11$ & $757 \pm 17$ & $1066 \pm 84$ & $855 \pm 23$ & \\
\hline 7649.935 & $5.38 \pm 0.39$ & $5.49 \pm 0.33$ & $3.90 \pm 0.27$ & $3.96 \pm 0.18$ & $0.92 \pm 0.20$ & $735 \pm 26$ & $1076 \pm 92$ & $846 \pm 35$ & $800 \pm 54$ \\
\hline 7653.850 & $5.69 \pm 0.26$ & $5.74 \pm 0.19$ & $4.23 \pm 0.18$ & $4.31 \pm 0.11$ & $1.06 \pm 0.11$ & $729 \pm 17$ & $943 \pm 65$ & $871 \pm 23$ & \\
\hline 7657.524 & $5.18 \pm 0.26$ & $5.26 \pm 0.16$ & $4.25 \pm 0.18$ & $4.04 \pm 0.10$ & $1.13 \pm 0.10$ & $707 \pm 17$ & $1076 \pm 83$ & $865 \pm 23$ & $854 \pm 25$ \\
\hline 7661.502 & $5.05 \pm 0.26$ & $5.17 \pm 0.19$ & $3.98 \pm 0.18$ & & $1.07 \pm 0.12$ & $682 \pm 17$ & $967 \pm 62$ & $834 \pm 23$ & $853 \pm 27$ \\
\hline 7665.495 & $5.26 \pm 0.26$ & $5.34 \pm 0.17$ & $3.78 \pm 0.18$ & $3.85 \pm 0.09$ & $0.93 \pm 0.11$ & $674 \pm 17$ & $924 \pm 71$ & $844 \pm 23$ & $799 \pm 35$ \\
\hline 7669.502 & $4.23 \pm 0.26$ & $4.29 \pm 0.16$ & $3.37 \pm 0.18$ & $3.42 \pm 0.10$ & $1.26 \pm 0.12$ & $655 \pm 17$ & $992 \pm 74$ & $818 \pm 23$ & $805 \pm 38$ \\
\hline 7669.599 & $4.28 \pm 0.26$ & $4.29 \pm 0.18$ & $3.54 \pm 0.18$ & $3.46=$ & $1.30 \pm$ & & $856 \pm 59$ & $836 \pm 23$ & \\
\hline 7673.551 & $4.20 \pm 0.26$ & $4.07 \pm 0.13$ & $3.36 \pm 0.18$ & $3.32 \pm$ & $1.33 \pm 0.11$ & $651 \pm 17$ & $885 \pm 55$ & $849 \pm 23$ & \\
\hline 7677.519 & $3.76 \pm 0.26$ & $3.62 \pm 0.15$ & $3.13 \pm 0.18$ & & $1.51 \pm 0.13$ & 613 & $2 \pm 62$ & $823 \pm 23$ & \\
\hline 7680.745 & $4.34 \pm 0.26$ & $4.29 \pm 0.19$ & $3.23 \pm 0.18$ & $3.16 \pm 0.10$ & $1.00 \pm 0.14$ & $592 \pm 17$ & & $805 \pm 23$ & $787 \pm 39$ \\
\hline 7680.852 & $3.60 \pm 0.39$ & $3.37 \pm 0.19$ & $3.08 \pm 0.27$ & $3.03 \pm 0.13$ & $1.65 \pm 0.19$ & $587 \pm 26$ & $824 \pm 69$ & $753 \pm 35$ & $772 \pm 48$ \\
\hline 7684.929 & $3.68 \pm 0.26$ & $3.63 \pm 0.13$ & $3.01 \pm 0.18$ & $2.95 \pm 0.08$ & & & $807 \pm 51$ & $780 \pm 23$ & \\
\hline 7685.019 & $3.03 \pm 0.39$ & $3.10 \pm 0.21$ & $2.55 \pm 0.27$ & $2.55 \pm 0.13$ & $1.36 \pm 0.23$ & & $674 \pm 56$ & $767 \pm 35$ & \\
\hline 7688.742 & $3.17 \pm 0.26$ & $3.14 \pm 0.14$ & $2.69 \pm 0.18$ & & $1.34 \pm 0.15$ & \pm 17 & $686 \pm 52$ & $723 \pm 23$ & \\
\hline 7688.840 & $2.89 \pm 0.26$ & $2.62 \pm 0.16$ & $2.46 \pm 0.18$ & $2.50 \pm 0.11$ & & & & $677 \pm 23$ & \\
\hline 7692.743 & $3.22 \pm 0.26$ & $3.05 \pm 0.14$ & $2.67 \pm 0.18$ & $2.59 \pm 0.09$ & $1.46 \pm 0.15$ & $492 \pm 17$ & $675 \pm 52$ & & \\
\hline 7692.843 & $3.24 \pm 0.26$ & $2.67 \pm 0.17$ & $2.71 \pm 0.18$ & & & $472 \pm 17$ & & $651 \pm 23$ & \\
\hline 7696.757 & $3.20 \pm 0.26$ & $3.30 \pm 0.11$ & $2.67 \pm 0.18$ & 2.73 & & $499 \pm 17$ & $655 \pm 47$ & $644 \pm 23$ & $675 \pm 33$ \\
\hline 7700.740 & $3.48 \pm 0.26$ & $3.57 \pm 0.13$ & $2.81 \pm 0.18$ & 2.81 & $1.22 \pm 0.12$ & $503 \pm 17$ & $646 \pm 44$ & $668 \pm 23$ & $652 \pm 26$ \\
\hline 7700.839 & $3.06 \pm 0.26$ & $3.23 \pm 0.19$ & $2.41 \pm 0.18$ & $2.51 \pm 0.11$ & $1.18 \pm 0.19$ & & & $690 \pm 23$ & $657 \pm 34$ \\
\hline 7705.430 & $3.56 \pm 0.26$ & $3.54 \pm 0.14$ & $2.76 \pm 0.18$ & $2.86 \pm 0.09$ & & & & $665 \pm 23$ & \\
\hline 7709.340 & $4.47 \pm 0.26$ & $4.51 \pm 0.15$ & $3.35 \pm 0.18$ & & $0.96 \pm 0$ & & $781 \pm 78$ & $699 \pm 23$ & $722 \pm 28$ \\
\hline 7713.335 & $4.41 \pm 0.26$ & $4 \pm 0.15$ & $3.25 \pm 0$ & & $1.06 \pm 0.11$ & $574 \pm 17$ & $806 \pm 56$ & $790 \pm 23$ & $755 \pm 27$ \\
\hline 7717.323 & 0.26 & $4.44 \pm 0.16$ & $3.42 \pm 0.18$ & $3.44 \pm 0.10$ & $1.16 \pm 0.12$ & $634 \pm 17$ & $852 \pm 53$ & $823 \pm 23$ & $781 \pm 29$ \\
\hline 7721.335 & $4.80 \pm 0.26$ & $4.72 \pm 0.19$ & $3.29 \pm 0.18$ & $3.35 \pm 0.10$ & $0.87 \pm 0.1$ & $611 \pm 17$ & $828 \pm 73$ & $856 \pm 23$ & $791 \pm 35$ \\
\hline 7725.337 & & & $2.91 \pm 0.18$ & $3.03 \pm 0.09$ & $0.99 \pm 0.13$ & $613 \pm 17$ & $785 \pm 64$ & $874 \pm 23$ & $868 \pm 32$ \\
\hline 7725.436 & $48 \pm 0.39$ & $3.22 \pm 0$. & $2.81 \pm 0.27$ & $2.74 \pm 0.14$ & $1.48 \pm 0.22$ & $600 \pm 26$ & $885 \pm 68$ & $810 \pm 35$ & $861 \pm 47$ \\
\hline 7729.670 & $2.81 \pm 0.26$ & $2.63 \pm 0.13$ & $2.37 \pm 0.18$ & $2.32 \pm 0.09$ & $1.59 \pm 0.16$ & $549 \pm 17$ & $787 \pm 57$ & $822 \pm 23$ & $799 \pm 24$ \\
\hline 7729.768 & $2.29 \pm 0.39$ & $2.61 \pm 0.25$ & $2.01 \pm 0.27$ & $1.91 \pm 0.14$ & $0.97 \pm 0.31$ & $538 \pm 26$ & $692 \pm 53$ & $773 \pm 35$ & $708 \pm 52$ \\
\hline 7733.680 & $2.21 \pm 0.26$ & $2.11 \pm 0.12$ & $1.98 \pm 0.18$ & $1.93 \pm 0.09$ & & & & $658 \pm 23$ & $646 \pm 24$ \\
\hline 7733.775 & $2.08 \pm 0.39$ & $2.19 \pm 0.24$ & $1.79 \pm 0.27$ & $1.58 \pm 0.13$ & $0.93 \pm 0.36$ & $453 \pm 26$ & $449 \pm 52$ & $558 \pm 35$ & $522 \pm 43$ \\
\hline 7737.662 & $1.68 \pm 0.26$ & $1.59 \pm 0.09$ & $1.60 \pm 0.18$ & $1.55 \pm 0.07$ & $1.90 \pm 0.18$ & $407 \pm 17$ & $523 \pm 40$ & $496 \pm 23$ & $514 \pm 26$ \\
\hline 7737.759 & $1.50 \pm 0.26$ & $1.52 \pm 0.13$ & $1.31 \pm 0.18$ & $1.35 \pm 0.09$ & $1.61 \pm 0.28$ & $397 \pm 17$ & $443 \pm 45$ & $526 \pm 23$ & $508 \pm 31$ \\
\hline 7741.582 & $1.73 \pm 0.26$ & $1.64 \pm 0.10$ & $1.70 \pm 0.18$ & $1.56 \pm 0.07$ & $1.83 \pm 0.20$ & $388 \pm 17$ & $503 \pm 32$ & $466 \pm 23$ & $469 \pm 26$ \\
\hline 7745.557 & $1.88 \pm 0.26$ & $1.77 \pm 0.09$ & $1.65 \pm 0.18$ & $1.67 \pm 0.06$ & $1.81 \pm 0.16$ & $396 \pm 17$ & $568 \pm 40$ & $475 \pm 23$ & $481 \pm 24$ \\
\hline
\end{tabular}

\footnotetext{
${ }^{a}$ Vignetting correction factors: $F_{1350}$ and $F_{1337}, 1.28 ; F_{1840}$ and $F_{1813}, 1.41 ; \mathrm{Ly} \alpha, 1.31 ; \mathrm{C}$ IV, 1.33
}

between the $\mathrm{He}$ II and the $\mathrm{C}$ IV lines. Because the C IV $\lambda 1549$ wings overwhelm those of $\mathrm{He}$ II $\lambda 1640$ in that range, the SIPS interval therefore underestimates the true extent of the He II feature. As pointed out by Wamsteker et al. (1990), the very broad wings of He II $\lambda 1640$ are particularly strong in NGC 5548 and dominate the total line emission. This explains why the GEX fits, which recover a fraction of the flux in the wing from the region where it is blended with C IV $\lambda 1549$, yield systematically larger He II intensities than SIPS.

\section{THE PATTERN OF VARIABILITY}

Before discussing the variability, we set out the mean properties of NGC 5548's ultraviolet spectrum as determined by our monitoring. The average line and continuum fluxes are listed in Table 6.

The average spectral index for the power-law fit to the SWP spectrum $\left(F_{v} \propto v^{-\alpha}\right)$ is $\langle\alpha\rangle \simeq 1.17$. Correction for Galactic reddening $\left(E_{B-V}=0.05\right.$; Wamsteker et al. 1990) brings this value to $\simeq 0.95$. The intrinsic continuum in NGC 5548 may be slightly flatter, however. As mentioned in $\S$ III $a$, blended lowcontrast Fe II lines create an artificial continuum whose intensity decreases rapidly with wavelength but which still contributes about $10 \%$ to the average flux at $1840 \AA$. After removal of the $\mathrm{Fe}$ II line spectrum and Balmer continuum emission, Wamsteker et al. (1990) found a mean spectral index $\langle\alpha\rangle \simeq 0.39$ for the NGC 5548 energy distribution, from the 
TABLE 3

WEAK EMISSION LiNES

\begin{tabular}{|c|c|c|c|c|c|c|c|}
\hline \multirow{2}{*}{$\begin{array}{c}\text { J.D. } \\
(-2,440,000)\end{array}$} & \multicolumn{2}{|c|}{ SiIV + OIV] $\lambda 1402$} & \multicolumn{2}{|c|}{$\operatorname{HeII} \lambda 1640^{a}$} & \multicolumn{2}{|c|}{ CIII] $\lambda 1909$} & \multirow{2}{*}{$\begin{array}{r}\mathrm{NV} \lambda 1240 \\
\text { GEX }\end{array}$} \\
\hline & & & $\begin{array}{l}\text { SIPS } \\
\quad(10\end{array}$ & GEX & SIPS & GEX & \\
\hline 7510.139 & $37 \pm 11$ & $45 \pm 7$ & $43 \pm 10$ & $58 \pm 19$ & $92 \pm 15$ & $74 \pm 8$ & $94 \pm 20$ \\
\hline 7514.082 & $50 \pm 11$ & $48 \pm 11$ & $64 \pm 10$ & $68 \pm 15$ & $116 \pm 15$ & $95 \pm 10$ & $104 \pm 25$ \\
\hline 7517.926 & $47 \pm 11$ & $42 \pm 7$ & $46 \pm 10$ & $88 \pm 22$ & $118 \pm 15$ & $90 \pm 8$ & $90 \pm 22$ \\
\hline 7522.091 & $65 \pm 11$ & $55 \pm 10$ & $61 \pm 10$ & $88 \pm 12$ & $117 \pm 15$ & & $<133 \pm 32$ \\
\hline 7526.099 & $45 \pm 11$ & $40 \pm 9$ & $74 \pm 10$ & $108 \pm 16$ & $118 \pm 15$ & $107 \pm 11$ & $82 \pm 30$ \\
\hline 7530.013 & $28 \pm 11$ & $42 \pm 8$ & $58 \pm 10$ & $97 \pm 15$ & $94 \pm 15$ & $84 \pm 10$ & $108 \pm 27$ \\
\hline & & & & $99 \pm 16$ & & & $82 \pm 27$ \\
\hline 753 & $38 \pm 11$ & $65 \pm 9$ & $65 \pm 10$ & & & & \\
\hline & $58 \pm 11$ & & $97 \pm 10$ & $133 \pm 16$ & $109 \pm 15$ & 10 & \pm 34 \\
\hline 7538.355 & $48 \pm 11$ & $62 \pm 11$ & $72 \pm 10$ & $120 \pm 14$ & $86 \pm 15$ & & $161 \pm 41$ \\
\hline 7543.214 & $37 \pm 11$ & $35 \pm 9$ & $76 \pm 10$ & $108 \pm 15$ & $113 \pm 15$ & $91 \pm 13$ & $132 \pm 30$ \\
\hline & & & & & & & \pm 33 \\
\hline & & & $93 \pm 10$ & & & & \pm 30 \\
\hline 7549.831 & $62 \pm$ & & $82 \pm 10$ & & $120 \pm 15$ & & \pm 23 \\
\hline 7553.160 & $74 \pm 11$ & $73 \pm 13$ & $81 \pm 10$ & $108 \pm 15$ & $112 \pm 15$ & $88 \pm 10$ & $107 \pm 33$ \\
\hline 7557.159 & $59 \pm 11$ & $79 \pm 12$ & $81 \pm 10$ & $74 \pm 12$ & $158 \pm 15$ & $109 \pm 10$ & $64 \pm 23$ \\
\hline 7557 & $49 \pm$ & & 0 & & & & \pm 34 \\
\hline 7561.855 & $42 \pm 11$ & & $42 \pm 10$ & $49 \pm 9$ & & & $63 \pm 23$ \\
\hline $7565.871^{b}$ & $56 \pm 11$ & $63 \pm 10$ & $44 \pm 10$ & $62 \pm 11$ & $153 \pm 15$ & $133 \pm 8$ & $109 \pm 25$ \\
\hline & $40 \pm 11$ & & $62 \pm 10$ & $68 \pm 8$ & $136 \pm 15$ & & $76 \pm 22$ \\
\hline & & & & & $136 \pm 15$ & $105 \pm 8$ & $4 \pm 17$ \\
\hline 7577.919 & $51 \pm$ & & 10 & $32 \pm 11$ & $106 \pm 15$ & & $37 \pm 30$ \\
\hline 7581.866 & $29 \pm 11$ & $27 \pm 7$ & $39 \pm 10$ & $36 \pm 6$ & $128 \pm 15$ & $106=$ & $9 \pm 18$ \\
\hline 7586.013 & $32 \pm 11$ & $34 \pm 6$ & $45 \pm 10$ & $49 \pm 6$ & $88 \pm 15$ & & $65 \pm 21$ \\
\hline 7586.107 & $43 \pm 17$ & $28 \pm 13$ & $69 \pm 15$ & & & & $39 \pm 22$ \\
\hline 7589 & & & & & & & \\
\hline 7590.089 & & & $45 \pm 10$ & $37 \pm 6$ & & & \pm 29 \\
\hline 7594.001 & $28 \pm 11$ & & $56 \pm 10$ & $55 \pm 6$ & $95 \pm 15$ & & $30 \pm 21$ \\
\hline & & & & $50 \pm 8$ & $113 \pm 15$ & \pm 11 & $96 \pm 37$ \\
\hline 7597.998 & $30 \pm 11$ & $34 \pm 12$ & $70 \pm 10$ & $63 \pm 7$ & $84 \pm 15$ & $68 \pm 9$ & $61 \pm 19$ \\
\hline 7598.090 & $50 \pm 11$ & $44 \pm 13$ & $76 \pm 10$ & $43 \pm 7$ & & & $7 \pm 40$ \\
\hline 7601.997 & & & & $66 \pm 7$ & $64 \pm 15$ & & $37 \pm 26$ \\
\hline 7602.095 & $45 \pm 11$ & & & $58 \pm 6$ & & & $92 \pm 27$ \\
\hline & & & & & & & $20 \pm 21$ \\
\hline 7606.103 & $37 \pm 17$ & $63 \pm 15$ & $31 \pm 15$ & $30 \pm 5$ & $124 \pm 23$ & $96 \pm 10$ & $139 \pm 42$ \\
\hline 7609.905 & & & $77 \pm 10$ & $89 \pm 8$ & $89 \pm 15$ & $97 \pm 10$ & $59 \pm 21$ \\
\hline 7613.685 & & & $72 \pm 10$ & $104 \pm 2$ & $105 \pm 15$ & $80 \pm 8$ & $106 \pm 28$ \\
\hline & & & $56 \pm 10$ & & $100 \pm 15$ & & $23 \pm 25$ \\
\hline & & & & & & & $181 \pm 32$ \\
\hline 7625.591 & $59 \pm 11$ & $73 \pm 11$ & $56 \pm 10$ & $97 \pm 5$ & $126 \pm 15$ & $96 \pm 9$ & $132 \pm 42$ \\
\hline 7629.740 & $52 \pm 11$ & & $70 \pm 10$ & $89 \pm 17$ & $117 \pm 15$ & $97 \pm 11$ & $120 \pm 38$ \\
\hline 7629.845 & $53 \pm 11$ & $74 \pm 21$ & $56 \pm 10$ & $75 \pm 5$ & $72 \pm 15$ & $86 \pm 13$ & $155 \pm 44$ \\
\hline 7633.926 & $63 \pm 11$ & & & & $105 \pm 15$ & & $100 \pm 38$ \\
\hline 7634.017 & $48 \pm 17$ & $31 \pm 19$ & $69 \pm 15$ & $52 \pm 6$ & $144 \pm 23$ & $136 \pm 18$ & $145 \pm 51$ \\
\hline 7637.916 & $55 \pm 11$ & $60 \pm 11$ & $79 \pm 10$ & $88 \pm 20$ & $122 \pm 15$ & $97 \pm 12$ & $82 \pm 37$ \\
\hline
\end{tabular}

optical to the ultraviolet. The contamination by Fe II lines is even greater in the LWP band, making it futile to attempt a description of the continuum shape there.

The ultraviolet spectrum of NGC 5548 varied significantly during the observing campaign. This is clearly illustrated by Figure 1, where the mean SWP spectrum during the local minimum of late February to early March is plotted on the same scale as the mean SWP spectrum 2 months later, when the continuum and line fluxes reached a maximum.

The light curves of the continuum flux, the FES, and the strong $C$ IV $\lambda 1549$ and $\operatorname{Ly} \alpha \lambda 1216$ emission lines are shown in Figure 3, whereas those of the other lines appear in Figure 4. Apart from the $\mathrm{N} v \lambda 1240$ line, these figures were generated from the SIPS measurements. It is obvious from these curves 
TABLE 3--Continued

\begin{tabular}{|c|c|c|c|c|c|c|c|}
\hline \multirow{2}{*}{$\begin{array}{c}\text { J.D. } \\
(-2,440,000)\end{array}$} & \multicolumn{2}{|c|}{ SiIV+OIV] $\lambda 1402$} & \multicolumn{2}{|c|}{$\mathrm{HeII} \lambda 1640^{\circ}$} & \multicolumn{2}{|c|}{ CIII] $\lambda 1909$} & \multirow{2}{*}{$\begin{array}{c}\text { NV } \lambda 1240 \\
\text { GEX }\end{array}$} \\
\hline & SIPS & GEX & $\begin{array}{l}\text { SIPS } \\
\qquad(10\end{array}$ & $\begin{array}{c}\text { GEX } \\
-14 \text { erg s }^{-1}\end{array}$ & SIPS & GEX & \\
\hline 7638.012 & $74 \pm 11$ & $82 \pm 19$ & $81 \pm 10$ & $88 \pm 5$ & $123 \pm 15$ & $106 \pm 14$ & $6 \pm 39$ \\
\hline 7641.981 & $52 \pm 11$ & $50 \pm 11$ & $93 \pm 10$ & $103 \pm 23$ & $112 \pm 15$ & $85 \pm 10$ & $100 \pm 34$ \\
\hline 7645.906 & $58 \pm 11$ & $66 \pm 14$ & $115 \pm 10$ & $113 \pm 4$ & $137 \pm 15$ & $90 \pm 11$ & $165 \pm 40$ \\
\hline 7645.993 & $52 \pm 11$ & $69 \pm 12$ & $76 \pm 10$ & $105 \pm 10$ & $142 \pm 15$ & $127 \pm 10$ & $86 \pm 48$ \\
\hline 7649.840 & $51 \pm 11$ & $56 \pm 10$ & $101 \pm 10$ & $115 \pm 6$ & $137 \pm 15$ & $106 \pm 12$ & $164 \pm 42$ \\
\hline 7649.935 & $72 \pm 17$ & $78 \pm 22$ & $83 \pm 15$ & $80 \pm 4$ & $121 \pm 23$ & $115 \pm 19$ & $272 \pm 50$ \\
\hline 7653.850 & $53 \pm 11$ & $69 \pm 11$ & $84 \pm 10$ & $119 \pm 10$ & $110 \pm 15$ & $103 \pm 12$ & $67 \pm 25$ \\
\hline 7657.524 & $71 \pm 11$ & $81 \pm 8$ & $81 \pm 10$ & $121 \pm 8$ & $126 \pm 15$ & $121 \pm 10$ & $153 \pm 31$ \\
\hline 7661.502 & $68 \pm 11$ & $92 \pm 11$ & $84 \pm 10$ & $137 \pm 10$ & $136 \pm 15$ & $150 \pm 18$ & $122 \pm 28$ \\
\hline 7665.495 & $61 \pm 11$ & $55 \pm 9$ & $70 \pm 10$ & $94 \pm 29$ & $127 \pm 15$ & $104 \pm 10$ & $97 \pm 32$ \\
\hline 7669.502 & $69 \pm 11$ & $61 \pm 10$ & $82 \pm 10$ & $107 \pm 2$ & $146 \pm 15$ & $133 \pm 11$ & $147 \pm 28$ \\
\hline 7669.599 & $88 \pm 11$ & $74 \pm 14$ & $77 \pm 10$ & $88 \pm 37$ & $133 \pm 15$ & $111 \pm 10$ & $37 \pm 29$ \\
\hline 7673.551 & $62 \pm 11$ & $74 \pm 10$ & $66 \pm 10$ & $88 \pm 6$ & $127 \pm 15$ & $115 \pm 10$ & $92 \pm 29$ \\
\hline 7677.519 & $66 \pm 11$ & $93 \pm 11$ & $71 \pm 10$ & $79 \pm 14$ & $137 \pm 15$ & $105 \pm 11$ & $144 \pm 27$ \\
\hline 7680.745 & $57 \pm 11$ & $58 \pm 10$ & $66 \pm 10$ & $63 \pm 2$ & $140 \pm 15$ & $126 \pm 12$ & $53 \pm 20$ \\
\hline 7680.852 & $30 \pm 17$ & $67 \pm 19$ & $90 \pm 15$ & $107 \pm 6$ & $152 \pm 23$ & $123 \pm 13$ & $85 \pm 33$ \\
\hline 7684.929 & $67 \pm 11$ & $67 \pm 8$ & $61 \pm 10$ & $70 \pm 5$ & $141 \pm 15$ & $126 \pm 10$ & $111 \pm 25$ \\
\hline 7685.019 & $69 \pm 17$ & $62 \pm 13$ & $48 \pm 15$ & $47 \pm 14$ & $153 \pm 23$ & $153 \pm 17$ & $105 \pm 29$ \\
\hline 7688.742 & $61 \pm 11$ & $66 \pm 10$ & $33 \pm 10$ & $42 \pm 5$ & $125 \pm 15$ & $117 \pm 10$ & $42 \pm 23$ \\
\hline 7688.840 & $47 \pm 11$ & $50 \pm 13$ & $55 \pm 10$ & $38 \pm 6$ & $149 \pm 15$ & $132 \pm 13$ & $56 \pm 31$ \\
\hline 7692.743 & $57 \pm 11$ & $72 \pm 10$ & $59 \pm 10$ & $46 \pm 4$ & $120 \pm 15$ & $101 \pm 9$ & $68 \pm 19$ \\
\hline 7692.843 & $39 \pm 11$ & $59 \pm 14$ & $37 \pm 10$ & $33 \pm 16$ & $129 \pm 15$ & $112 \pm 15$ & $98 \pm 29$ \\
\hline 7696.757 & $44 \pm 11$ & $54 \pm 9$ & $37 \pm 10$ & $46 \pm 24$ & $112 \pm 15$ & $98 \pm 9$ & $41 \pm 25$ \\
\hline 7700.740 & $36 \pm 11$ & $34 \pm 8$ & $48 \pm 10$ & $53 \pm 2$ & $105 \pm 15$ & $92 \pm 8$ & $30 \pm 23$ \\
\hline 7700.839 & $65 \pm 11$ & $66 \pm 13$ & $39 \pm 10$ & $57 \pm 32$ & $137 \pm 15$ & $118 \pm 11$ & $66 \pm 23$ \\
\hline 7705.430 & $45 \pm 11$ & $59 \pm 9$ & $56 \pm 10$ & $65 \pm 9$ & $127 \pm 15$ & $100 \pm 10$ & $75 \pm 23$ \\
\hline 7709.340 & $40 \pm 11$ & $51 \pm 9$ & $58 \pm 10$ & $78 \pm 5$ & $112 \pm 15$ & $102 \pm 10$ & $75 \pm 27$ \\
\hline 7713.335 & $66 \pm 11$ & $70 \pm 10$ & $71 \pm 10$ & $97 \pm 7$ & $146 \pm 15$ & $106 \pm 10$ & $101 \pm 28$ \\
\hline 7717.323 & $51 \pm 11$ & $57 \pm 9$ & $86 \pm 10$ & $109 \pm 40$ & $121 \pm 15$ & $100 \pm 11$ & $101 \pm 25$ \\
\hline 7721.335 & $54 \pm 11$ & $58 \pm 8$ & $81 \pm 10$ & $75 \pm 2$ & $105 \pm 15$ & $108 \pm 14$ & $94 \pm 27$ \\
\hline 7725.337 & $50 \pm 11$ & $75 \pm 9$ & $72 \pm 10$ & $105 \pm 23$ & $140 \pm 15$ & $108 \pm 10$ & $45 \pm 23$ \\
\hline 7725.436 & $66 \pm 17$ & $85 \pm 17$ & $74 \pm 15$ & $102 \pm 6$ & $131 \pm 23$ & $126 \pm 20$ & $139 \pm 42$ \\
\hline 7729.670 & $60 \pm 11$ & $67 \pm 10$ & $62 \pm 10$ & $62 \pm 7$ & $116 \pm 15$ & $107 \pm 8$ & $52 \pm 28$ \\
\hline 7729.768 & $44 \pm 17$ & $43 \pm 18$ & $63 \pm 15$ & $55 \pm 25$ & $144 \pm 23$ & $140 \pm 17$ & $71 \pm 27$ \\
\hline 7733.680 & $56 \pm 11$ & $72 \pm 11$ & $33 \pm 10$ & $28 \pm 4$ & $135 \pm 15$ & $113 \pm 25$ & $62 \pm 24$ \\
\hline 7733.775 & $23 \pm 17$ & $28 \pm 14$ & $39 \pm 15$ & $38 \pm 42$ & $137 \pm 23$ & $135 \pm 18$ & $110 \pm 38$ \\
\hline 7737.662 & $32 \pm 11$ & $41 \pm 7$ & $35 \pm 10$ & $22 \pm 1$ & $126 \pm 15$ & $99 \pm 8$ & $0 \pm 18$ \\
\hline 7737.759 & $43 \pm 11$ & $42 \pm 10$ & $52 \pm 10$ & $26 \pm 23$ & $129 \pm 15$ & $116 \pm 10$ & $53 \pm 21$ \\
\hline 7741.582 & $43 \pm 11$ & $50 \pm 8$ & $24 \pm 10$ & $27 \pm 2$ & $82 \pm 15$ & $80 \pm 7$ & $40 \pm 14$ \\
\hline 7745.557 & $33 \pm 11$ & $48 \pm 7$ & $30 \pm 10$ & $18 \pm 7$ & $112 \pm 15$ & $81 \pm 6$ & $18 \pm 18$ \\
\hline
\end{tabular}

that significant changes were observed in all these quantities, and that the dominant time scale of variation (i.e., the "pseudoperiod") was $\sim 80$ days.

Table 6 summarizes the main characteristics of the variability for each of the quantities we measured: its mean value over the entire period of observations, the fractional variation $F_{\text {var }}$ defined as the ratio of the rms fluctuation to the mean flux, and the ratio of maximum to minimum flux, $R_{\max }$. The fractional variations have been corrected to represent the intrinsic variations by subtracting the mean measuring error for the feature in quadrature. Except for $\mathrm{N} v \lambda 1240$, both the SIPS and the
GEX values are listed. Again, the agreement between the two methods is excellent, there being no systematic differences apart from those already noted which affect the Ly $\alpha \lambda 1240$ and He II $\lambda 1640$ fluxes.

The longer the wavelength, the smaller the amplitude of the continuum variations. $F_{\text {var }}$ decreases gradually from $\simeq 0.30$ at $1350 \AA$ to 0.26 at $1840 \AA, 0.16$ at $2670 \AA$, and 0.08 near $5000 \AA$ (FES). Since the variations are simultaneous in all three bands (see Table 7), this implies that the spectrum becomes systematically bluer when it gets brighter. The large dilution of the nuclear continuum by stellar emission accounts for most of the 
TABLE 4

LWP RESULTS

\begin{tabular}{|c|c|c|c|c|}
\hline \multirow{2}{*}{$\begin{array}{c}\text { JD } \\
(-2,440,000) \\
\end{array}$} & \multicolumn{2}{|c|}{$F_{2670^{a}}$} & \multicolumn{2}{|c|}{$\operatorname{Mg}$ II $\lambda 2798^{b}$} \\
\hline & SIPS & GEX & SIPS & GEX \\
\hline $\begin{array}{l}7510.085 \ldots \ldots \ldots \\
7514.150 \ldots \ldots \ldots \\
7517.994 \ldots \ldots \ldots \\
7522.158 \ldots \ldots \ldots \\
7526.168 \ldots \ldots \ldots\end{array}$ & $\begin{array}{l}2.33 \pm 0.19 \\
2.46 \pm 0.16 \\
2.47 \pm 0.16 \\
2.66 \pm 0.21 \\
2.76 \pm 0.29\end{array}$ & $\begin{array}{l}2.24 \pm 0.11 \\
2.41 \pm 0.13 \\
2.43 \pm 0.14 \\
2.64 \pm 0.16 \\
2.73 \pm 0.18\end{array}$ & $\begin{array}{l}137 \pm 6 \\
123 \pm 6 \\
134 \pm 6 \\
134 \pm 6 \\
131 \pm 6\end{array}$ & $\begin{array}{l}123 \pm 7 \\
125 \pm 8 \\
132 \pm 8 \\
123 \pm 9 \\
119 \pm 9\end{array}$ \\
\hline $\begin{array}{l}7530.087 \ldots \ldots \ldots \\
7534.072 \ldots \ldots \ldots \\
7538.238 \ldots \ldots \ldots \\
7543.265 \ldots \ldots \ldots \\
7546.078 \ldots \ldots \ldots\end{array}$ & $\begin{array}{l}2.62 \pm 0.17 \\
2.69 \pm 0.15 \\
2.94 \pm 0.17 \\
3.00 \pm 0.21 \\
3.02 \pm 0.25\end{array}$ & $\begin{array}{l}2.64 \pm 0.15 \\
2.68 \pm 0.12 \\
2.88 \pm 0.13 \\
2.93 \pm 0.14 \\
3.10 \pm 0.16\end{array}$ & $\begin{array}{l}137 \pm 6 \\
138 \pm 6 \\
145 \pm 6 \\
131 \pm 6 \\
143 \pm 6\end{array}$ & $\begin{array}{l}125 \pm 8 \\
141 \pm 8 \\
147 \pm 9 \\
129 \pm 8 \\
135 \pm 8\end{array}$ \\
\hline $\begin{array}{l}7549.898 \ldots \ldots \ldots \\
7553.208 \ldots \ldots \ldots \\
7557.207 \ldots \ldots \ldots \\
7561.923 \ldots \ldots \ldots \\
7563.249 \ldots \ldots \ldots\end{array}$ & $\begin{array}{l}2.78 \pm 0.16 \\
2.83 \pm 0.23 \\
2.64 \pm 0.15 \\
2.27 \pm 0.23 \\
2.10 \pm 0.25\end{array}$ & $\begin{array}{l}2.73 \pm 0.12 \\
2.68 \pm 0.15 \\
2.53 \pm 0.14 \\
2.30 \pm 0.14 \\
2.01 \pm 0.14\end{array}$ & $\begin{array}{l}151 \pm 6 \\
130 \pm 6 \\
139 \pm 6 \\
130 \pm 6 \\
151 \pm 6\end{array}$ & $\begin{array}{l}137 \pm 8 \\
130 \pm 8 \\
135 \pm 8 \\
137 \pm 8 \\
150 \pm 8\end{array}$ \\
\hline $\begin{array}{l}7565.931^{\mathrm{c}} \ldots \ldots \ldots \\
7569.919 \ldots \ldots \ldots \\
7573.790 \ldots \ldots \ldots \\
7577.879 \ldots \ldots \ldots \\
7581.935 \ldots \ldots \ldots\end{array}$ & $\begin{array}{l}2.27 \pm 0.27 \\
2.23 \pm 0.16 \\
1.88 \pm 0.22 \\
1.90 \pm 0.17 \\
1.96 \pm 0.29\end{array}$ & $\begin{array}{l}2.14 \pm 0.17 \\
2.13 \pm 0.13 \\
1.95 \pm 0.15 \\
1.82 \pm 0.11 \\
1.89 \pm 0.15\end{array}$ & $\begin{array}{l}148 \pm 6 \\
148 \pm 6 \\
152 \pm 6 \\
136 \pm 6 \\
130 \pm 6\end{array}$ & $\begin{array}{l}153 \pm 10 \\
131 \pm 8 \\
141 \pm 8 \\
136 \pm 7 \\
124 \pm 7\end{array}$ \\
\hline $\begin{array}{l}7585.990 \ldots \ldots \ldots \\
7590.052 \ldots \ldots \ldots \\
7594.063 \ldots \ldots \ldots \\
7598.054 \ldots \ldots \ldots \\
7602.054 \ldots \ldots \ldots\end{array}$ & $\begin{array}{l}2.08 \pm 0.16 \\
2.16 \pm 0.22 \\
2.22 \pm 0.22 \\
2.29 \pm 0.14 \\
2.36 \pm 0.13\end{array}$ & $\begin{array}{l}1.95 \pm 0.14 \\
1.99 \pm 0.12 \\
2.07 \pm 0.14 \\
2.19 \pm 0.13 \\
2.34 \pm 0.11\end{array}$ & $\begin{array}{l}143 \pm 6 \\
127 \pm 6 \\
118 \pm 6 \\
122 \pm 6 \\
124 \pm 6\end{array}$ & $\begin{array}{l}138 \pm 9 \\
115 \pm 7 \\
128 \pm 7 \\
134 \pm 8 \\
114 \pm 6\end{array}$ \\
\hline $\begin{array}{l}7606.062 \ldots \ldots \ldots \\
7613.755 \ldots \ldots \ldots \\
7617.565 \ldots \ldots \ldots \\
7621.657 \ldots \ldots \ldots \\
7625.660 \ldots \ldots \ldots\end{array}$ & $\begin{array}{l}2.41 \pm 0.12 \\
2.73 \pm 0.13 \\
2.78 \pm 0.13 \\
3.25 \pm 0.17 \\
3.23 \pm 0.20\end{array}$ & $\begin{array}{l}2.38 \pm 0.14 \\
2.68 \pm 0.13 \\
2.80 \pm 0.15 \\
3.23 \pm 0.15 \\
3.22 \pm 0.16\end{array}$ & $\begin{array}{l}120 \pm 6 \\
134 \pm 6 \\
129 \pm 6 \\
146 \pm 6 \\
143 \pm 6\end{array}$ & $\begin{array}{l}128 \pm 8 \\
134 \pm 8 \\
123 \pm 10 \\
133 \pm 8 \\
142 \pm 10\end{array}$ \\
\hline $\begin{array}{l}7629.803 \ldots \ldots \ldots \\
7633.979 \ldots \ldots \ldots \\
7637.969 \ldots \ldots \ldots \\
7642.032 \ldots \ldots \ldots \\
7645.956 \ldots \ldots \ldots\end{array}$ & $\begin{array}{l}3.17 \pm 0.15 \\
3.19 \pm 0.18 \\
2.99 \pm 0.24 \\
3.37 \pm 0.19 \\
3.11 \pm 0.24\end{array}$ & $\begin{array}{l}3.07 \pm 0.13 \\
3.13 \pm 0.13 \\
3.05 \pm 0.15 \\
3.16 \pm 0.16 \\
3.05 \pm 0.15\end{array}$ & $\begin{array}{l}128 \pm 6 \\
133 \pm 6 \\
133 \pm 6 \\
132 \pm 6 \\
128 \pm 6\end{array}$ & $\begin{array}{l}116 \pm 8 \\
126 \pm 7 \\
128 \pm 10 \\
128 \pm 9 \\
122 \pm 8\end{array}$ \\
\hline $\begin{array}{l}7649.894 \ldots \ldots \ldots \\
7653.901 \ldots \ldots \ldots \\
7657.579 \ldots \ldots \ldots \\
7661.564 \ldots \ldots \ldots \\
7665.569 \ldots \ldots \ldots\end{array}$ & $\begin{array}{l}3.09 \pm 0.16 \\
2.93 \pm 0.17 \\
2.87 \pm 0.16 \\
2.73 \pm 0.19 \\
2.78 \pm 0.13\end{array}$ & $\begin{array}{l}3.06 \pm 0.13 \\
2.90 \pm 0.15 \\
2.94 \pm 0.14 \\
2.71 \pm 0.15 \\
2.74 \pm 0.12\end{array}$ & $\begin{array}{l}131 \pm 6 \\
140 \pm 6 \\
148 \pm 6 \\
138 \pm 6 \\
146 \pm 6\end{array}$ & $\begin{array}{l}131 \pm 7 \\
139 \pm 10 \\
140 \pm 9 \\
116 \pm 8 \\
143 \pm 8\end{array}$ \\
\hline $\begin{array}{l}7669.551 \ldots \ldots \ldots \\
7673.508 \ldots \ldots \ldots \\
7677.577 \ldots \ldots \ldots \\
7680.810 \ldots \ldots \ldots \\
7684.887 \ldots \ldots \ldots\end{array}$ & $\begin{array}{l}2.61 \pm 0.16 \\
2.59 \pm 0.17 \\
2.43 \pm 0.14 \\
2.49 \pm 0.17 \\
2.28 \pm 0.17\end{array}$ & $\begin{array}{l}2.60 \pm 0.14 \\
2.55 \pm 0.14 \\
2.36 \pm 0.13 \\
2.44 \pm 0.13 \\
2.22 \pm 0.12\end{array}$ & $\begin{array}{l}146 \pm 6 \\
140 \pm 6 \\
151 \pm 6 \\
142 \pm 6 \\
152 \pm 6\end{array}$ & $\begin{array}{l}149 \pm 10 \\
126 \pm 9 \\
152 \pm 9 \\
139 \pm 8 \\
153 \pm 8\end{array}$ \\
\hline $\begin{array}{l}7684.988 \ldots \ldots \ldots \\
7688.801 \ldots \ldots \ldots \ldots \\
7692.801 \ldots \ldots \ldots \ldots \\
7696.815 \ldots \ldots \ldots \ldots \\
7700.798 \ldots \ldots \ldots\end{array}$ & $\begin{array}{l}2.25 \pm 0.20 \\
2.13 \pm 0.15 \\
2.13 \pm 0.13 \\
2.14 \pm 0.16 \\
2.15 \pm 0.17\end{array}$ & $\begin{array}{l}2.18 \pm 0.15 \\
2.07 \pm 0.14 \\
2.11 \pm 0.14 \\
2.04 \pm 0.12 \\
2.09 \pm 0.13\end{array}$ & $\begin{array}{l}155 \pm 6 \\
152 \pm 6 \\
150 \pm 6 \\
143 \pm 6 \\
144 \pm 6\end{array}$ & $\begin{array}{l}144 \pm 9 \\
155 \pm 8 \\
137 \pm 8 \\
144 \pm 8 \\
131 \pm 9\end{array}$ \\
\hline $\begin{array}{l}7705.491 \ldots \ldots \ldots \\
7709.403 \ldots \ldots \ldots \\
7713.396 \ldots \ldots \ldots \\
7717.379 \ldots \ldots \ldots \\
7721.403 \ldots \ldots \ldots\end{array}$ & $\begin{array}{l}2.24 \pm 0.17 \\
2.38 \pm 0.17 \\
2.50 \pm 0.10 \\
2.58 \pm 0.15 \\
2.48 \pm 0.16\end{array}$ & $\begin{array}{l}2.19 \pm 0.14 \\
2.25 \pm 0.11 \\
2.41 \pm 0.12 \\
2.54 \pm 0.15 \\
2.47 \pm 0.12\end{array}$ & $\begin{array}{l}145 \pm 6 \\
144 \pm 6 \\
143 \pm 6 \\
144 \pm 6 \\
146 \pm 6\end{array}$ & $\begin{array}{l}138 \pm 10 \\
134 \pm 7 \\
142 \pm 8 \\
142 \pm 9 \\
143 \pm 7\end{array}$ \\
\hline $\begin{array}{l}7725.392 \ldots \ldots \ldots \\
7729.729 \ldots \ldots \ldots \\
7733.736 \ldots \ldots \ldots \\
7737.721 \ldots \ldots \ldots \\
7741.656 \ldots \ldots \ldots\end{array}$ & $\begin{array}{l}2.30 \pm 0.14 \\
2.00 \pm 0.16 \\
1.78 \pm 0.16 \\
1.62 \pm 0.17 \\
1.47 \pm 0.14\end{array}$ & $\begin{array}{l}2.30 \pm 0.13 \\
1.96 \pm 0.11 \\
1.65 \pm 0.10 \\
1.57 \pm 0.10 \\
1.41 \pm 0.10\end{array}$ & $\begin{array}{l}139 \pm 6 \\
142 \pm 6 \\
154 \pm 6 \\
148 \pm 6 \\
144 \pm 6\end{array}$ & $\begin{array}{l}136 \pm 7 \\
144 \pm 6 \\
149 \pm 5 \\
136 \pm 7 \\
137 \pm 7\end{array}$ \\
\hline $7745.620 \ldots \ldots \ldots$ & $1.49 \pm 0.13$ & $1.46 \pm 0.11$ & $141 \pm 6$ & $125 \pm 6$ \\
\hline
\end{tabular}

a In units of $10^{-14} \mathrm{ergs} \mathrm{s}^{-1} \mathrm{~cm}^{-2} \AA^{-1}$.

b In units of $10^{-14} \mathrm{ergs} \mathrm{s}^{-1} \mathrm{~cm}^{-2}$.

c Vignetting correction factors: $F_{2670}, 1.21 ; \mathrm{Mg}$ II, 1.20 .

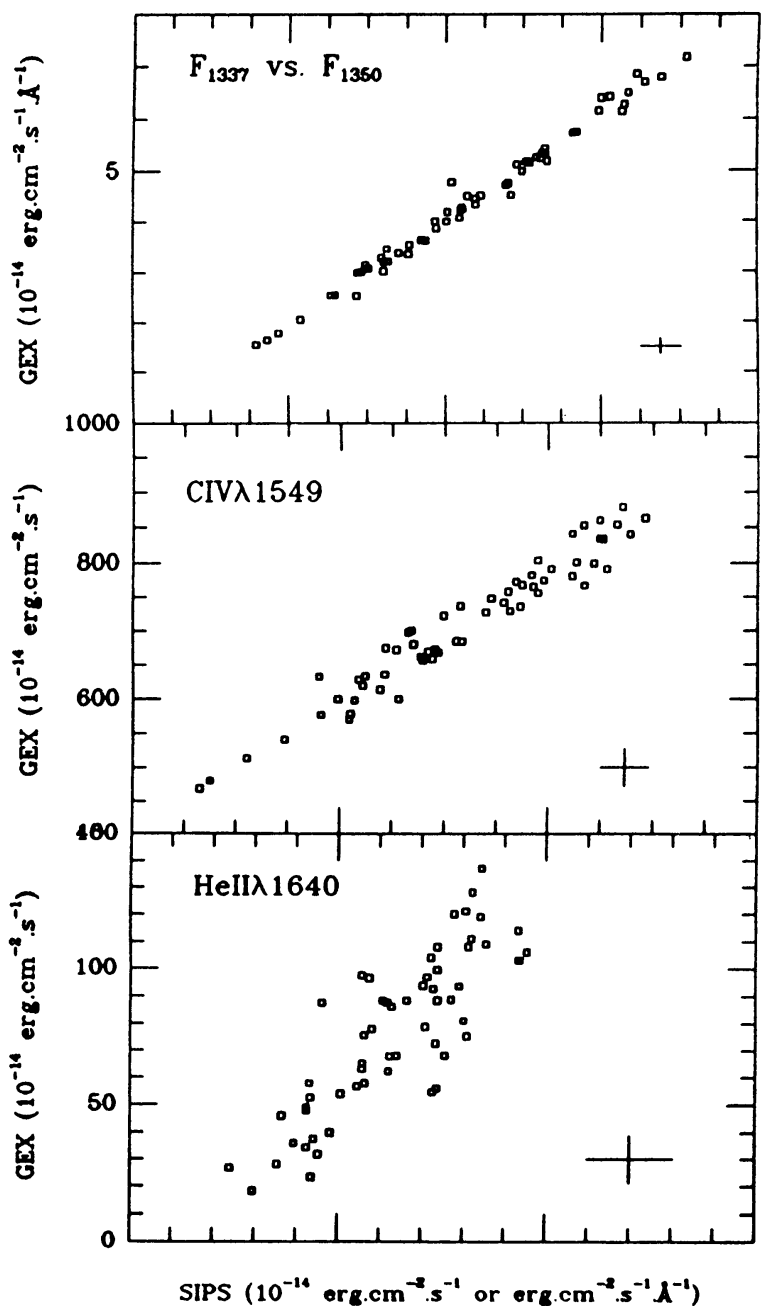

FIG. 2.-Flux, as obtained with GEX, plotted as a function of the SIPS flux for the continuum near $1300 \AA$ (upper panel), the C IV $\lambda 1549$ emission line (middle panel), and the $\mathrm{He}$ II $\lambda 1640+\mathrm{O}$ III] $\lambda 1663$ blend (lower panel). A typical error bar is shown in the lower right-hand corner of each panel. The scale is such that the range covered is the same along the $X$-and the $Y$-axis.

reduced amplitude of the variations in the optical. Much of the diminution in variability at the longer ultraviolet wavelengths is probably due to dilution by relatively steady $\mathrm{Fe}$ II and Balmer continuum emission. In the SWP range, the full range of spectral index variations is $\Delta \alpha=1.1 \pm 0.2$, with rms fluctuations $\Delta \alpha=0.23$. Blended Fe II lines-provided that their intensity does not vary more than that of $\mathrm{Mg}$ II $\lambda 2798$ - account for variations of $\sim 0.3$ in the spectral index if their contribution amounts to $10 \%$ of the $1840 \AA$ continuum flux when it is minimum. The remaining change in $\alpha$ could be due to genuine spectral variations of the underlying continuum. Wamsteker $e t$ al. (1990), after taking into account the Fe II and Bac contribution, also find it necessary to allow for some variability (albeit small) of the optical to ultraviolet spectral index in NGC 5548, the continuum becoming systematically bluer when it brightens. A more precise estimate of the amplitude of the spectral variations will be possible when the present data are combined with the optical spectra recorded almost simultaneously (Peterson et al. 1990a).

All the emission-line fluxes also varied significantly. However, the amplitude of these variations differs greatly from one line to the next. The high-ionization lines $N$ v $\lambda 1240$ and 
TABLE 5

GEX VERSUS SIPS LINEAR REGRESSION

\begin{tabular}{|c|c|c|c|c|c|}
\hline Feature & Slope & Intercept $^{\mathbf{a}}$ & $r$ & $\chi_{v}^{2}$ & $P\left(\geq \chi_{v}^{2}\right)$ \\
\hline$F_{1350 / 1337} \ldots \ldots \ldots \ldots \ldots \ldots$ & $1.048 \pm 0.026$ & $-0.17 \pm 0.12$ & 0.995 & 0.308 & $>0.999$ \\
\hline$F_{1840 / 1813} \cdots \cdots \cdots \cdots \cdots$ & $1.010 \pm 0.026$ & $-0.00 \pm 0.09$ & 0.994 & 0.336 & $>0.999$ \\
\hline$F_{2670} \ldots \ldots \ldots \ldots \ldots \ldots$ & $1.022 \pm 0.064$ & $-0.11 \pm 0.16$ & 0.992 & 0.060 & $>0.999$ \\
\hline $\mathrm{Ly} \alpha+\mathrm{N} v$ & $1.533 \pm 0.062$ & $-96 \pm 37$ & 0.957 & 0.620 & 0.989 \\
\hline C IV $\lambda 1549 \ldots \ldots$ & $0.949 \pm 0.029$ & $29 \pm 30$ & 0.975 & 0.455 & $>0.999$ \\
\hline $\mathrm{Mg}$ II $\lambda 2798 \ldots \ldots$ & $0.998 \pm 0.010$ & $-5 \pm 3$ & 0.736 & 0.506 & $>0.999$ \\
\hline C III] $\lambda 1909 \ldots \ldots$. & $0.868 \pm 0.081$ & $-3 \pm 2$ & 0.782 & 0.448 & $>0.999$ \\
\hline $\mathrm{He} \mathrm{II}+\mathrm{O}$ III $] \ldots .$. & $1.950 \pm 0.102$ & $-50 \pm 14$ & 0.827 & 0.979 & 0.520 \\
\hline Si IV + O Iv $] \ldots$ & $1.212 \pm 0.184$ & $-3 \pm 9$ & 0.688 & 0.604 & 0.992 \\
\hline
\end{tabular}

${ }^{a}$ Same units as in Tables 2-4.

TABLE 6

VARIABILITY PARAMETERS

\begin{tabular}{|c|c|c|c|c|c|c|}
\hline \multirow[b]{2}{*}{ Feature } & \multicolumn{2}{|c|}{ Mean Flux ${ }^{a}$} & \multicolumn{2}{|c|}{$F_{\text {var }}$} & \multicolumn{2}{|c|}{$R_{\max }$} \\
\hline & SIPS & GEX & SIPS & GEX & SIPS & GEX \\
\hline$F_{1350 / 1337} \ldots \ldots \ldots \ldots \ldots$ & 4.36 & 4.39 & 0.30 & 0.32 & $4.46 \pm 0.53$ & $4.64 \pm 0.29$ \\
\hline$F_{1840 / 1813} \ldots \ldots \ldots \ldots \ldots$ & 3.31 & 3.33 & 0.26 & 0.27 & $3.45 \pm 0.46$ & $3.35 \pm 0.16$ \\
\hline$F_{2670} \ldots \ldots \ldots \ldots \ldots \ldots$ & 2.49 & 2.44 & 0.16 & 0.17 & $2.30 \pm 0.25$ & $2.29 \pm 0.20$ \\
\hline $\operatorname{Ly} \alpha+N v \ldots \ldots \ldots \ldots$ & 556. & 760. & 0.17 & 0.17 & $1.93 \pm 0.09$ & $2.14 \pm 0.21$ \\
\hline $\mathrm{C}_{\text {IV }} \lambda 1549 \ldots \ldots \ldots \ldots$ & 718. & 708. & 0.14 & 0.13 & $1.92 \pm 0.11$ & $1.87 \pm 0.12$ \\
\hline Mg II $\lambda 2798 \ldots \ldots \ldots \ldots$ & 139. & 134. & 0.05 & 0.04 & $1.31 \pm 0.08$ & $1.36 \pm 0.10$ \\
\hline $\mathrm{He} \mathrm{II}+\mathrm{O} \mathrm{III}] \ldots \ldots \ldots \ldots$ & 64.0 & 77.8 & 0.23 & 0.30 & $3.96 \pm 1.70$ & $7.57 \pm 3.02$ \\
\hline C III] $\lambda 1909 \ldots \ldots \ldots \ldots$ & 118. & 100. & 0.09 & 0.11 & $1.86 \pm 0.28$ & $2.02 \pm 0.36$ \\
\hline $\mathrm{Si}$ IV $+\mathrm{O}$ IV $] \ldots \ldots \ldots \ldots$ & 49.4 & 57.3 & 0.15 & 0.19 & $2.98 \pm 1.30$ & $3.49 \pm 1.02$ \\
\hline $\mathrm{N} v \lambda 1240 \ldots \ldots \ldots \ldots \ldots$ & $\ldots$ & 89.7 & $\ldots$ & 0.49 & $\ldots$ & $56 \pm 243$ \\
\hline FES $\ldots \ldots \ldots \ldots \ldots \ldots$ & \multicolumn{2}{|c|}{50.6} & \multicolumn{2}{|c|}{0.08} & \multicolumn{2}{|c|}{$1.37 \pm 0.03$} \\
\hline
\end{tabular}

a Same units as in Tables 2-4.

He II $\lambda 1640$ had variations whose amplitude was as great as or greater than that of the short-wavelength continuum, with $F_{\text {var }} \simeq 0.5$ and 0.23 , respectively. Lines of intermediate ionization had lower amplitudes, with $F_{\text {var }}$ ranging from 0.09 to 0.17 for C IV $\lambda 1549, \mathrm{C}$ III] $\lambda 1909$, Ly $\alpha \lambda 1216$, and $\mathrm{Si}$ IV $+\mathrm{O}$ IV] $\lambda 1402$. The lowest ionization line in our data set, $\mathrm{Mg}$ II $\lambda 2798$, exhibited the lowest amplitude fluctuations, with $F_{\text {var }} \simeq 0.05$. Because significant variations were found in all the emission lines, detailed structural analysis should be possible for all of them.

\section{TIME SERIES ANALYSIS}

The variations took the form of two large-amplitude "events," plus an additional slightly lower amplitude event near the end of the observing campaign. Here an event is defined as a statistically significant local maximum in the light

TABLE 7

RESULTS FROM CROSS-CORRELATIONS

\begin{tabular}{|c|c|c|c|c|}
\hline Feature & $\begin{array}{c}\Delta t(\text { Peak) } \\
\text { (days) }\end{array}$ & $\begin{array}{c}\Delta t(\text { Center) } \\
\text { (days) }\end{array}$ & Amplitude & $\begin{array}{c}\text { FWHM } \\
\text { (days) }\end{array}$ \\
\hline$F_{1840 / 1813} \ldots \ldots \ldots \ldots$ & 0 & 0 & 0.98 & 38 \\
\hline$F_{2670} \cdots \cdots \ldots \ldots \ldots$ & 0 & 0 & 0.97 & 40 \\
\hline FES $\ldots \ldots \ldots \ldots \ldots$ & 2 & 4 & 0.88 & 42 \\
\hline $\mathrm{N} v \lambda 1240 \ldots \ldots \ldots$ & 4 & 4 & 0.79 & 34 \\
\hline $\mathrm{He} \mathrm{II}+\mathrm{O} \mathrm{III}] \ldots \ldots$. & 4 & 10 & 0.67 & 40 \\
\hline $\mathrm{C}$ iv $\lambda 1549 \ldots \ldots \ldots$ & 8 & 16 & 0.66 & 38 \\
\hline $\operatorname{Ly} \alpha+N \mathrm{~N} \ldots \ldots \ldots$ & 12 & 12 & 0.84 & 34 \\
\hline $\mathrm{Si}$ IV $+\mathrm{O}$ IV $] \ldots \ldots . .$. & 12 & 34 & 0.61 & 36 \\
\hline C III] $\lambda 1909 \ldots \ldots \ldots$ & 26 & 32 & 0.56 & 40 \\
\hline $\operatorname{Mg}$ II $\lambda 2798 \quad \ldots \ldots \ldots$ & \multicolumn{2}{|c|}{$\simeq 34-72$} & 0.46 & 72 \\
\hline
\end{tabular}

curve followed and preceded by a local minimum. Each individual event lasted typically $\simeq 80-100$ days, except the last one, whose duration was only about 40 days. This is in any case much larger than the interval between two consecutive observations. Hence, our temporal resolution is adequate to characterize the main ultraviolet variability of NGC 5548. Moreover, the sampling of the light curves is almost perfectly even, the mean (and error on the mean) interval between two observations being $3.99 \pm 0.04$ days. On top of the long-term trend are superposed a few episodes of rapid, small-amplitude variability, e.g., the dip near JD 2,447,534-most conspicuous in the light curves of Ly $\alpha, \mathrm{C}$ IV, and the FES-or the "shoulder" between JD 2,447,606 and 2,447,621.

The overall resemblance of the continuum band and emission-line light curves is striking. All three major events are clearly recognizable in most light curves. There are some noticeable differences, however. For instance, during the second event, the $1350 \AA$ continuum flux rose more rapidly than the $\operatorname{Ly} \alpha \lambda 1216$ or C IV $\lambda 1549$ emission-line flux and reached its maximum 10-15 days earlier. This suggests that the variations of the lines are delayed with respect to those of the ultraviolet continuum, as predicted by the photoionization models discussed in $\S \mathbf{I}$.

To quantify this delay, we computed the cross-correlation between the emission lines and the $1350 \AA$ (and $1337 \AA$ ) continuum, as first suggested by Gaskell and Sparke (1986). Unlike previous work in this area, whose uneven sampling required either interpolation (as reviewed by Peterson 1988a) or the discrete correlation method (Edelson and Krolik 1988), our data allowed use of the conventional method. That is, at all lags $\tau$ which are integral multiples of our 4 day sampling inter- 


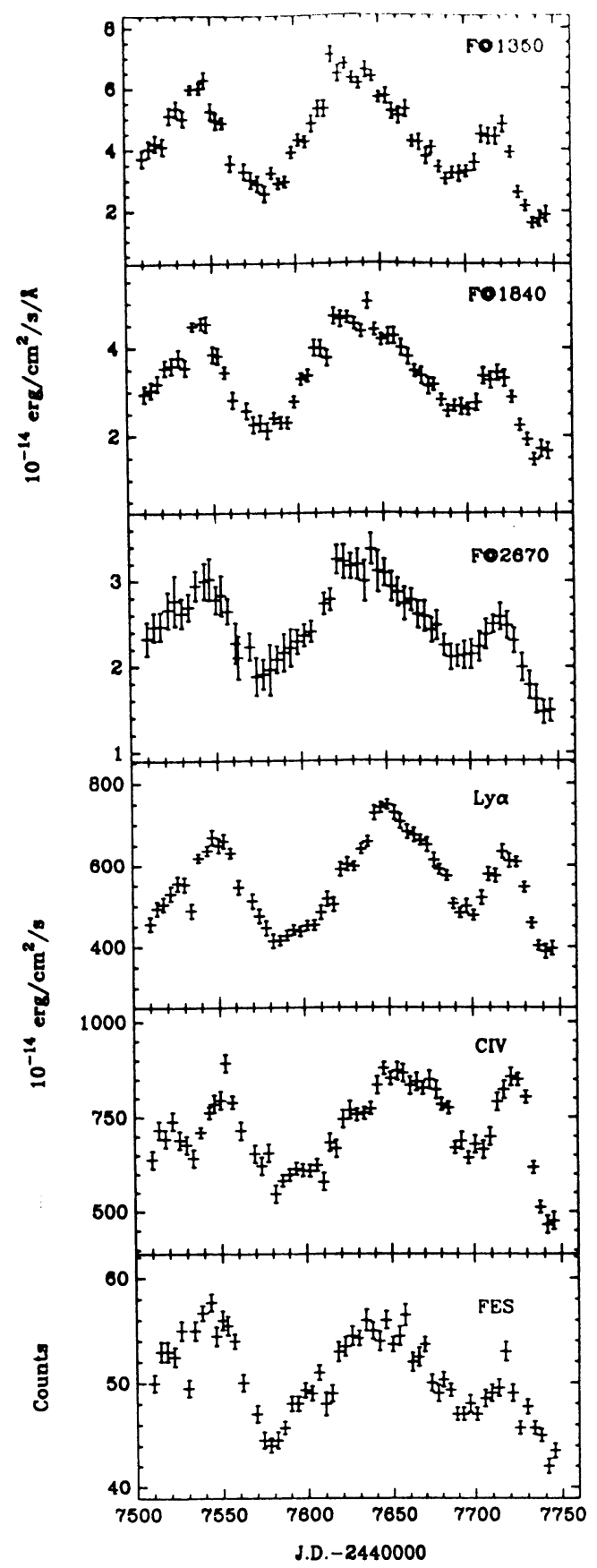

Fig. 3.-Top to bottom: Light curves of the continuum at 1350, 1840 and $2670 \AA$; the Ly $\alpha \lambda 1216$ and C IV $\lambda 1549$ emission lines; and the optical light curve as measured with the FES. Units are $10^{-14} \mathrm{ergs} \mathrm{cm}^{-2} \mathrm{~s}^{-1} \AA^{-1}$ for the continuum fluxes, $10^{-14} \mathrm{ergs} \mathrm{cm}^{-2} \mathrm{~s}^{-1}$ for the lines, and counts for the FES measurements. The fluxes are represented with their associated errors. Notice the large amplitude of the variations and the similarity of the light curves.

val, we computed the mean value of

$$
\left[F_{l}\left(t_{i}\right)-\bar{F}_{l}\right]\left[F_{c}\left(t_{i}-\tau\right)-\bar{F}_{c}\right] /\left(\sigma_{l} \sigma_{c}\right),
$$

where $F_{l, c}$ is the (line, continuum) flux, and $\sigma_{l, c}$ is the standard deviation about the mean flux $\bar{F}_{l, c}$. The results are displayed in Table 7 and in Figures 5 and 6. Note that the SIPS and GEX values yield identical results. It is immediately apparent that the peak amplitude of the cross-correlation is substantial in all cases. Only in the case of $\mathrm{Mg}$ II $\lambda 2798$ does the correlation amplitude at the peak fall below 0.5 . This fact gives very strong support to the long-held view that the ionizing continuum is the source of energy for the emission lines.

The uncertainty on the location of the maximum of these cross-correlations arises from two sources: measurement error and incomplete sampling. To assess the importance of measurement errors, we have performed a Monte Carlo simulation. Artificial light curves were generated by randomly assigning a statistically probable flux value at each epoch, that is, a value which falls within the range allowed by the measure-

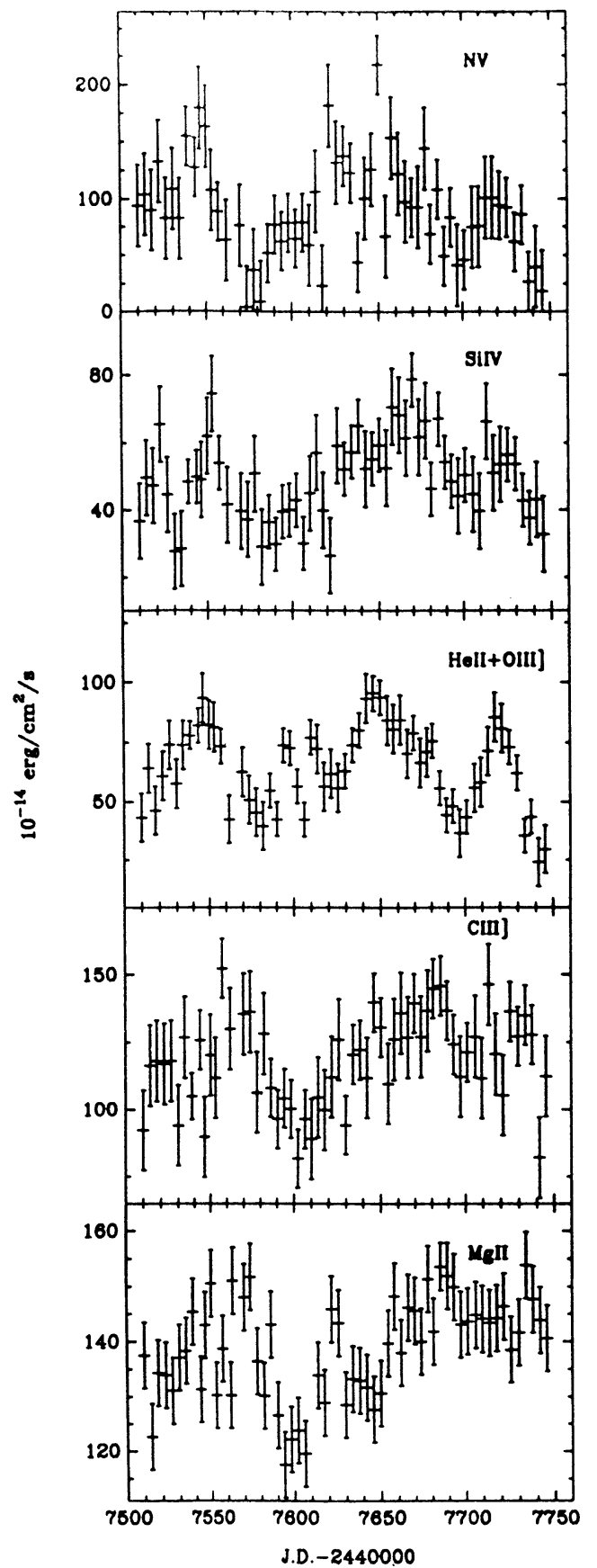

FIG. 4.-Same as Fig. 3, but for the weaker $N v \lambda 1240$, Si IV + O Iv] $\lambda 1402$, $\mathrm{He}$ II $\lambda 1640(+\mathrm{O}$ III] $\lambda 1663), \mathrm{C} \mathrm{III]} \lambda 1909$, and $\mathrm{Mg}$ II $\lambda 2798$ emission lines. 


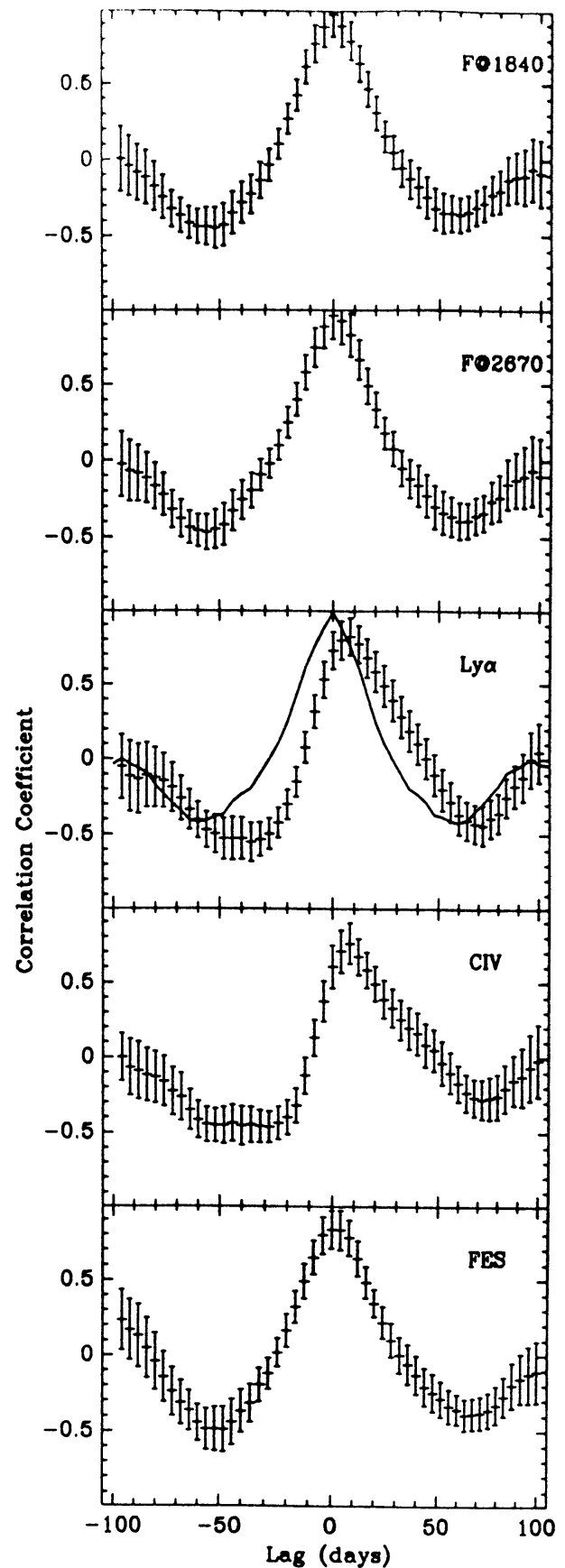

FIG. 5.-Cross-correlations (CCs) of the different emission lines and continua with the continuum flux at $1350 \AA$. Top to bottom: CC of the continuum at 1840 and at $2670 \AA$, of the $\operatorname{Ly} \alpha \lambda 1216$ and $C$ IV $\lambda 1549$ emission lines, and of the FES. The points are shown with their associated errors calculated as in Edelson and Krolik (1988). For reference, the autocorrelation function of the $1350 \AA$ continuum is shown in the panel in the middle (fully drawn line). Note the large value of the correlation coefficients at the peak and the very small delays of the emission lines.

ment and its associated uncertainty. Flux values are selected in a such way that their distribution about the mean is normal. Then, each light curve is correlated with a corresponding light curve of the $1350 \AA$ continuum obtained in the same way. The whole process is reiterated a large number of times, and the distribution of the locations of the cross-correlation peak is analyzed. The standard deviation of these distributions gives a reliable estimate of the uncertainty on the lag due to measurement errors. This uncertainty turns out to be small: $\epsilon(\Delta t)= \pm 0.13$ and \pm 0.17 days for the strong $\operatorname{Ly} \alpha \lambda 1216$ and $\mathrm{C}$ IV $\lambda 1549$ lines, respectively, while the weaker and noiser He II $\lambda 1640$ feature yields $\epsilon(\Delta t)= \pm 1.8$ days.

Unfortunately, there is no universally accepted prescription for estimating the larger uncertainty due to incomplete sam-

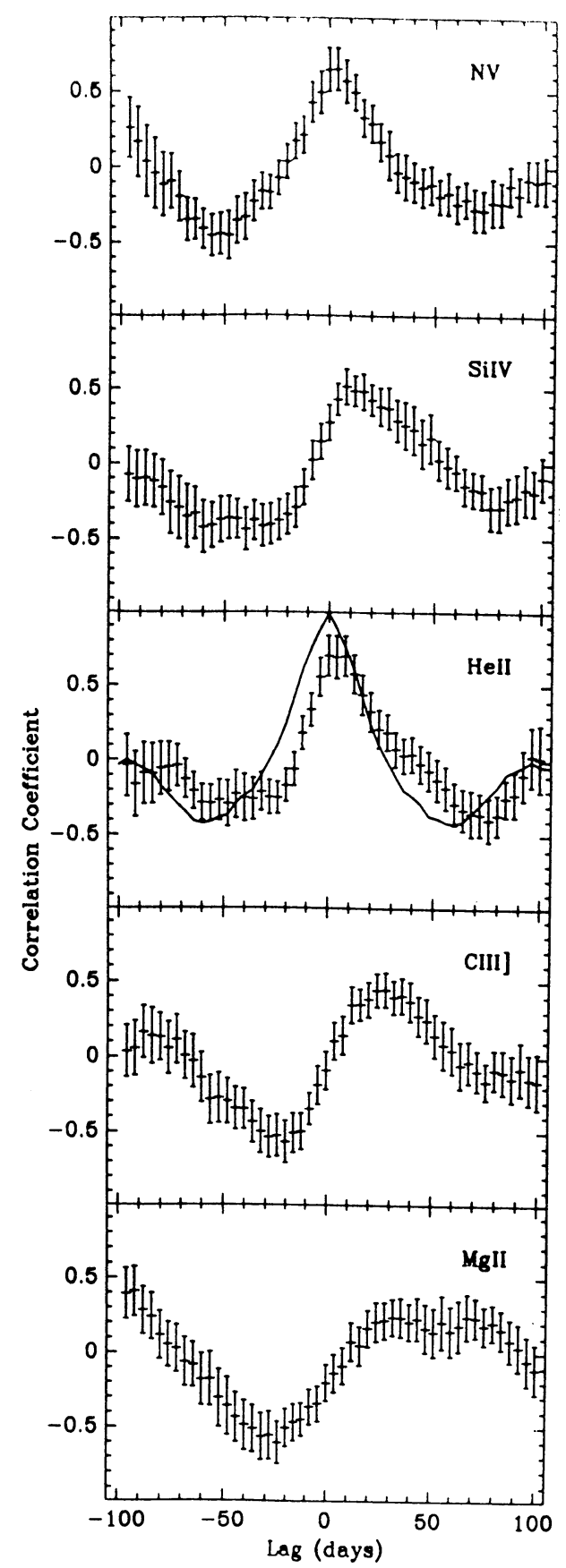

Fig. 6.-Same as Fig. 5, but for the weaker $N$ v $\lambda 1240$, Si Iv + O Iv] $\lambda 1402$, He II $\lambda 1640$ ( $+\mathrm{O}$ III] $\lambda 1663$ ), C III] $\lambda 1909$, and $\mathrm{Mg}$ II $\lambda 2798$ emission lines. Note the difference between the $\mathrm{He}$ II $\lambda 1640$ line, which is well correlated at very short lags with the $1350 \AA$ continuum, and $\mathrm{Mg}$ II $\lambda 2798$, for which the peak of the cross-correlation is much lower and displaced toward large positive lags. 
pling. Several ways to obtain such errors, and to assign to them a physical meaning, are discussed by Gaskell and Peterson (1987), Edelson and Krolik (1988), and Maoz and Netzer (1989). This lack of a well-defined error estimate complicates any attempt to make quantitative use of these cross-correlation functions. An additional complication arises because the continuum autocorrelation is quite broad and smooth: consequently, the cross-correlations are, also. To illustrate this difficulty, we have listed in Table 7 the position of the peak of the cross-correlations as well as the position of their "center." By "center" we mean the average of the two lags at which the cross-correlation passes through a level 0.3 below the peak. Taken together, these two give an order-of-magnitude estimate of the characteristic delay time scale. Any difference between them indicates the degree of skewness in the cross-correlation. The FWHM of the cross-correlation function can, in principle, provide another estimate of the response time scale. If the line emission can be described as a linear convolution of the continuum light curve with a response function, the cross-correlation function is the convolution of the continuum autocorrelation function with the response function. Any difference between the FWHM of the line-continuum cross-correlation and the continuum autocorrelation would then indicate the width of the response function.

The most significant result that may be derived from Table 7 is that, apart from $\mathrm{Mg}$ II 22798 , all the emission lines correlate extremely well with $F_{1350}$, and that the characteristic delays are quite short for most of them. There is also a general tendency for the highest ionization lines to have the shortest delays. For both He II $\lambda 1640$ and $N$ v $\lambda 1240$, the bulk of the cross-correlation is found in the neighborhood of 4-10 days, while for $C$ IV $\lambda 1549$ and $\operatorname{Ly} \alpha \lambda 1216$ the range is $8-16$ days. Si IV $\lambda 1400$ and C III] $\lambda 1909$ have cross-correlations concentrated between 12 and 34 days, while $\mathrm{Mg}$ II $\lambda 2798$ is found from 34 to 72 days. Less significance may be attached to the fact that Ly $\alpha, N$ v $\lambda 1240$, and Mg II $\lambda 2798$ have fairly symmetric crosscorrelation functions, while the cross-correlation functions for C IV $\lambda 1549, \mathrm{He}$ II $\lambda 1640$, and Si IV $\lambda 1400$ are quite skew, with the "centers" falling at lags much larger than the peaks. In essentially all cases, the cross-correlation function (as measured by FWHM) is not much wider than the continuum autocorrelation; in other words, only rough upper limits on the widths of the response functions can be placed by this comparison.

Finally, it is worth noting that, within the uncertainties, the continuum variations occur simultaneously in all four bands, from $1350 \AA$ to $\simeq 5000 \AA$.

\section{SUMMARY AND CONCLUSION}

The nucleus of NGC 5548 has been observed every four days during 8 months with the $I U E$ satellite. Significant variations of the emission lines and continuum flux took place. The amplitude of the variations is much larger than the measurement errors. This, together with the fact that the time scale for variability was an order of magnitude large than our sampling interval (i.e., the variations are time-resolved), allows us to draw firm and important conclusions concerning the pattern of variability of the ultraviolet (and optical) spectrum of NGC 5548.

1. The typical amplitude of the continuum variability decreases with increasing wavelength: $F_{\text {var }}=0.30,0.26,0.16$, and 0.08 for $\lambda \simeq 1350,1840,2700$, and $5000 \AA$, respectively. Because all the bands are correlated at zero lag, the continuum is systematically bluer when it is brighter. In the optical, most if not all of this effect can be attributed to dilution by stellar light. While the strong Fe II emission lines which contaminate the long-wavelength continuum and respond only very weakly to fluctuations in the continuum (Wamsteker et al. 1990) could account for much of the spectral variation in the nearultraviolet, the changes in the spectral index observed in the SWP may, at least in part, represent a genuine property of the underlying continuum.

2. The continuum variations appear to be simultaneous in all four ultraviolet and optical bands, i.e., from $\simeq 1300 \AA$ to $5000 \AA$.

3. The amplitude of variation of a given emission line seems to depend on the degree of ionization of its ion. The highest ionization lines show the strongest variations, with $N$ v $\lambda 1240$ and $\mathrm{He}$ II $\lambda 1640$ exhibiting maximum-to-minimum flux ratios as high as those of the continuum. Intermediate-ionization lines, including Ly $\alpha \lambda 1216, \mathrm{C}$ IV $\lambda 1549$, and C III] $\lambda 1909$, had maximum to minimum amplitudes of $\simeq 2$. $\mathrm{Mg}$ II 22798 , the lowest ionization line studied, exhibited the smallest amplitude fluctuations, $\simeq 1.3$.

4. Apart from Mg II 22798 , the emission-line variations correlate extremely well with those of the $1350 \AA$ continuum if allowance is made for a systematic short delay, lending qualitative support to the view that photoionization by the nuclear continuum is responsible for driving the emission lines. For $\mathrm{Mg}$ II the correlation is significantly weaker and the delay much longer than for other lines.

5. The delay of a given emission line seems to depend on the degree of ionization of its ion. $\mathrm{N} v \lambda 1240$ and He II $\lambda 1640$ exhibit the smallest delay, the bulk of their cross-correlation being in the neighborhood of 4-10 days. The delay for the intermediate-ionization $\operatorname{Ly} \alpha \lambda 1216$ and $C$ IV $\lambda 1549$ lines is somewhat larger, 8-16 days. The cross-correlations of $\mathrm{Si}$ IV + O IV] $\lambda 1402$ and C III] $\lambda 1909$ peak in the range 12-34 days, while that of $\mathrm{Mg}$ II $\lambda 2798$ is concentrated at lags of from $\simeq 34$ to 72 days. For comparison, a preliminary analysis of the optical data by Peterson et al. (1990a) shows that the maximum of the cross-correlation for the $\mathrm{H} \beta$ line occurs at a lag of $\simeq 24$ days.

These results set new and strong constraints on existing and future models of the broad emission line region in active galaxies and quasars, and in particular on the geometry and the ionization structure of the BLR.

It is a pleasure to thank the $I U E$ time allocation committees on both sides of the Atlantic, since, without their endorsement, this project would never have been feasible. We also want to express our deepest thanks to the schedulers at both IUE observatories for having performed the seemingly impossible task of scheduling the 60 IUE observations at a 4 day interval. The support of the resident astronomers at both $I U E$ observatories is also acknowledged. Finally, we wish to thank the following individuals who strongly supported the project but felt their contribution was not sufficient to have their name on the author's list: Tom Balonek (Hamilton), David Band (Lawrence Livermore), Jill Bechtold (Steward Observatory), Mitch Begelman (JILA), Catherine Boisson (Meudon), Ross Cohen (CASS), Stephen Cota (Aerospace Corporation), Thierry Courvoisier (Genève), David Crampton (DAO), Mike De Robertis (York University), Françoise Durret (IAP), Sperello Di Serego (ST-ECF), Richard Green (KPNO), John Hutchings (DAO), Timothy Kallman (NASA GSFC), Gordon 
MacAlpine (Ann Arbor), Laura Maraschi (Bologna), Shingo Nakatani (UCL), Daniel Péquignot (Meudon), Mike Penston (RGO), Andy Pollock (Birmingham), Almudena Prieto (Madrid), Andy Robinson (IoA), John Michael Shull (JILA),
Hartmut Schulz (Bochum), Alex Smith (University of Florida), Wayne Stein (Minneapolis), Roberto Terlevich (RGO), Megg Urry (STScI), Martin Ward (IoA), Mark Whittle (Charlottesville), and Bev and Derek Wills (Austin).
Andrillat, Y., and Souffrin, S. 1968, Ap. Letters, 1,111.

Avni, Y. 1976, Ap. J., 210, 642.

Barr, P., Willis, A. J., and Wilson, R. 1983, M.N.R.A.S., 203, 201

Barylak, M., Wasatonic, R., and Imhoff, C. 1984, ESA IUE Newsletter, 20, 201

Blandford, R. D., and McKee, C. F. 1982, Ap. J., 255, 419.

Boggess, A., et al. 1978 a, Nature, 275, 372 .

Boggess, A., et al. 1978b, Nature, 275, 377.

Bohlin, R. 1988, NASA IUE Newsletter, 34, 10

Bohlin, R. C., Holm, A. V., Savage, B. D., Snijders, M. A. J., and Sparks, W. M. 1980, Astr. Ap., 85, 1.

Branduardi-Raymont, G. 1988, in IAU Symposium 134, Active Galactic Nuclei, ed. D. E. Osterbrock and J. S. Miller (Dordrecht: Kluwer), p. 177.

Capriotti, E. R., Foltz, C. B., and Peterson, B. M. 1982, Ap. J., 261, 35

Cassatella, A., Barbero, J., and Benvenuti, P. 1985, Astr. Ap., 144, 335 (CBB).

Cherepashchuk, A. M., and Lyutyi, V. M. 1973, Ap. Letters, 13, 165.

Clavel, J., et al. 1987, Ap. J., 321, 251.

Clavel, J., Wamsteker, W., and Glass, I. S. 1989, Ap. J., 337, 236

Davidson, K., and Netzer, H. 1979, Rev. Mod. Phys., 51, 715.

Dibai, E. A., and Pronik, V. I. 1967, Astr. Zh., 44, 952.

Edelson, R. A., and Krolik, J. H. 1988, Ap. J., 333, 646

Edelson, R. A., and Malkan, M. A. 1986, Ap. J., 308, 59

Faelker, J., Gordon, F., and Sandford, M. C. W. 1987, in Exploring the Universe with the IUE Satellite, ed. Y. Kondo et al. (Dordrecht: Reidel), p. 21.

Fitch, W., Pacholczyk, A. G., and Weymann, R. J. 1967, Ap. J. (Letters), 150 L67.

Gaskell, C. M., and Peterson, B. M. 1987, Ap. J. Suppl., 65, 1.

Gaskell, C. M., and Sparke, L. S. 1986, Ap. J., 305, 175 .

Gregory, S., Ptak, R., and Stoner, R. 1982, Ap.J., 261, 30.

Harris, A. W., and Sonneborn, G. 1987, in Exploring the Universe with the IUE Satellite, ed. Y. Kondo et al. (Dordrecht: Reidel), p. 729.

Holm, A. V., and Crabb, W. G. 1979, NASA IUE Newsletter, 7, 40

\section{REFERENCES}

Holm, A. V., and Rice, G. 1981, NASA IUE Newsletter, 15, 74.

Kaastra, J. S., and Barr, P. 1989, Astr. Ap., 226, 59.

Koratkar, A. P., and Gaskell, C. M. 1989, Ap. J., 345, 637.

Maoz, D., and Netzer, H. 1989, M.N.R.A.S., 236, 21.

Maoz, D., et al. 1990, Ap. J., 351, 75.

Munoz-Peiro, J. R. 1985, NASA IUE Newsletter, $27,27$.

Netzer, H., et al. 1990, Ap. J., 353, 108.

Netzer, H., and Wills, B. J. 1983, Ap. J., 275, 445.

Peterson, B. M. 1988a, Pub. A.S.P., 100, 18. . 1988b, I AU Circ., No. 4664.

Peterson, B. M., Korista, K. T., and Wagner, R. M. 1989, A.J.,98, 500.

Peterson, B. M., et al. 1990a, in Proc. Conf. on Variability of Active Galactic Nuclei (Atlanta), ed. H. R. Miller and P. J. Wiita (Cambridge: Cambridge University Press).

Peterson, B. M., Reichert, G. A., Korista, K. T., and Wagner, R. M. 1990b, Ap. $J, 352,68$.

Reichert, G. A., and Kriss, G. 1990, in preparation

Rosenblatt, E. I., and Malkan, M. A. 1990, Ap. J., 350, 132.

Sonneborn, G., Oliversen, N. A., Imhoff, C. L., Pitts, R. E., and Holm, A. V. 1987, NASA IUE Newsletter, 32, 6.

Turner, T. J., and Pounds, K. 1989, M.N.R.A.S., 240, 833.

Turnrose, B. E., and Thompson, R. W. 1984, IUE Image Processing Information Manual, Version 2.0, CSC/TM-84/6058.

Ulrich, M. H., et al. 1984, M.N.R.A.S., 206, 221.

Urry, M., and Reichert, G. 1988, NASA IUE Newsletter, 34, 95.

Wamsteker, W., et al. 1990, Ap. J., 354, 446.

Wills, B. J., Netzer, H., and Wills, D. 1985, Ap. J., 248, 472.

Wilson, A. S., and Ulvestad, J. S. 1982, Ap. J., $260,56$.

Wilson, A. S., Wu, X., Bald win, J. A., and Balick, B. 1989, Ap. J., 339, 729.

York, D. 1966, Canadian J. Phys., 44, 1079. 